\title{
RhoBTB1 protects against hypertension and arterial stiffness by restraining phosphodiesterase 5 activity
}

\author{
Masashi Mukohda, ${ }^{1}$ Shi Fang, ${ }^{1,2}$ Jing Wu, ${ }^{1,2}$ Larry N. Agbor, ${ }^{1}$ Anand R. Nair, ${ }^{1}$ Stella-Rita C. Ibeawuchi, ${ }^{1}$ Chunyan Hu, ${ }^{1}$ Xuebo Liu, ${ }^{1}$ \\ Ko-Ting Lu, ${ }^{1,2}$ Deng-Fu Guo, ${ }^{1}$ Deborah R. Davis, ${ }^{1}$ Henry L. Keen, ${ }^{1}$ Frederick W. Quelle, ${ }^{1}$ and Curt D. Sigmund ${ }^{1,2}$ \\ 'Department of Pharmacology, UIHC Center for Hypertension Research, Roy J. and Lucille A. Carver College of Medicine, University of lowa, lowa City, lowa, USA. 2Department of Physiology, Cardiovascular \\ Center, Medical College of Wisconsin, Milwaukee, Wisconsin, USA.
}

\begin{abstract}
Mice selectively expressing a PPAR $\gamma$ dominant-negative mutation in vascular smooth muscle exhibit RhoBTB1 deficiency and hypertension. Our rationale was to use genetic complementation to uncover the mechanism of action of RhoBTB1 in vascular smooth muscle. Inducible smooth muscle-specific restoration of RhoBTB1 fully corrected hypertension and arterial stiffness by improving vasodilator function. Notably, the cardiovascular protection occurred despite the preservation of increased agonist-mediated contraction and RhoA and Rho kinase activity, suggesting that RhoBTB1 selectively controls vasodilation. RhoBTB1 augmented the cyclic $3^{\prime}, 5^{\prime}$-monophosphate (cCMP) response to NO by restraining the activity of phosphodiesterase 5 (PDE5) through its action as a substrate adaptor delivering PDE5 to the Cullin-3 E3 ring ubiquitin ligase complex for ubiquitination, thereby inhibiting PDE5. Angiotensin II infusion also caused RhoBTB1 deficiency and hypertension, which were prevented by smooth muscle-specific RhoBTB1 restoration. We conclude that RhoBTB1 protected against hypertension, vascular smooth muscle dysfunction, and arterial stiffness in at least 2 models of hypertension.
\end{abstract}

\section{Introduction}

PPAR $\gamma$ is a ligand-activated transcription factor known to regulate fatty acid metabolism. The clinical significance of PPAR $\gamma$ is best illustrated by the improvement in glycemic control in patients with type 2 diabetes treated with thiazolidinediones (TZDs), which are synthetic PPAR $\gamma$ agonists (1). The PROactive (PROspective pioglitAzone Clinical Trial In macroVascular Events) trial demonstrated that pioglitazone lowered arterial blood pressure (BP) and improved vascular function in type 2 diabetes (2), a finding confirmed by a number of other smaller trials and clinical studies (3-5). Although serious adverse effects of TZDs have been reported, whether TZDs are associated with adverse effects remains very controversial. The FDA initially restricted the use of some TZDs on the basis of reports showing an increased risk of myocardial infarction compared with other type 2 diabetes treatment regimens (6). That decision was reversed in 2013, when a review of the evidence including the RECORD (Rosiglitazone Evaluated for Cardiac Outcomes and Regulation of Glycemia in Diabetes) trial suggested that there was no increased overall risk of cardiovascular morbidity or mortality compared with standard glucose-lowering drugs $(7,8)$. Despite this, new prescriptions for the TZD class of drugs continue to decline $(9,10)$. Thus, identification of PPAR $\gamma$ target genes is required to design new therapies that selectively preserve the protective actions of PPAR $\gamma$, while eliminating the potential adverse effects of TZDs.

The best evidence supporting a role for PPAR $\gamma$ in BP regulation comes from genetic studies showing that mutations in PPAR $\gamma$

Conflict of interest: The authors have declared that no conflict of interest exists. Copyright: () 2019, American Society for Clinical Investigation.

Submitted: July 9, 2018; Accepted: March 19, 2019; Published: May 6, 2019.

Reference information: J Clin Invest. 2019;129(6):2318-2332.

https://doi.org/10.1172/JCI123462. cause human hypertension (11-14). Other studies suggested that the beneficial effect of PPAR $\gamma$ may be mediated through the vasculature (15). On the basis of the clinical and genetic literature, we hypothesized that loss of PPAR $\gamma$ function in tissues such as liver, muscle, and fat causes type 2 diabetes, whereas hypertension is caused by a loss of PPAR $\gamma$ activity in the blood vessel. This hypothesis was confirmed by studies of transgenic mice, in which targeting the expression of the human hypertension-causing mutations in PPAR $\gamma$ selectively to either endothelium or vascular smooth muscle was done (16-18). PPAR $\gamma$ also was found to have antiinflammatory effects on endothelium and smooth muscle $(19,20)$ as well as antioxidant effects on endothelium $(21,22)$.

Mechanistically, the expression of dominant-negative PPAR $\gamma$ mutations (P467L or V290M) selectively in vascular smooth muscle caused vascular dysfunction and hypertension that were dependent on increased RhoA and ROCK (Rho kinase) signaling $(16,23)$. Gene expression profiling of aortae from S-P467L mice led us to identify RhoBTB1 as one of approximately 250 genes altered by dominant-negative PPAR $\gamma$. RhoBTB1 is an atypical member of the Rho GTPase family and a member of the family of the Broad complex, Tramtrack, and Bric à brac (BTB) domaincontaining proteins (24). BTB domain-containing proteins are notable for their activity as substrate adaptors for the Cullin-3 E3 RING ubiquitin ligase complex (CRL3) (25). RhoBTB1 has been shown to bind Cullin-3 $(23,26)$, and we provided compelling evidence that RhoBTB1 is a PPAR $\gamma$ target gene in smooth muscle cells (SMCs) (23). This identification of RhoBTB1 as a PPAR $\gamma$ target and its connection with the CRL3 complex suggested that Cullin-3 may play a role in regulating vasomotor function. Consistent with this, Cullin-3 mediates RhoA turnover $(27,28)$. S-P467L mice exhibit impaired levels of Cullin-3, impaired turnover of RhoA, and increased RhoA/ROCK activity in response 
A

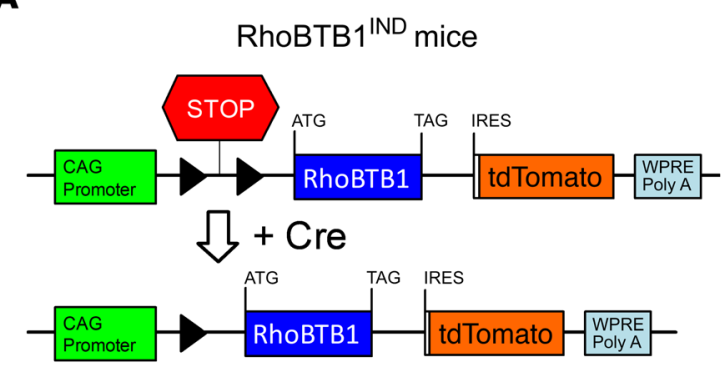

B

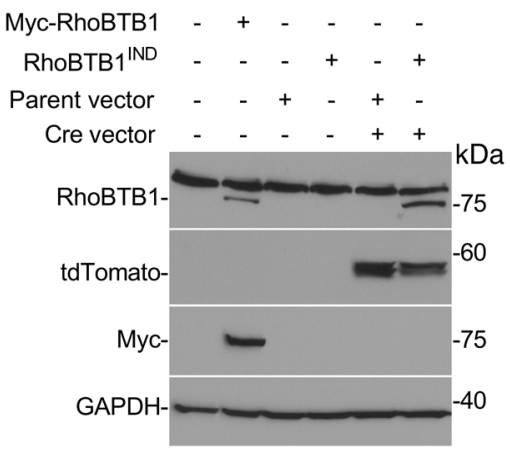

C

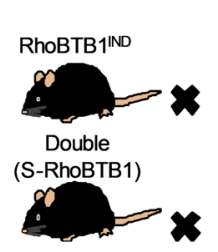
SMC-CreER T2 (Control)

E

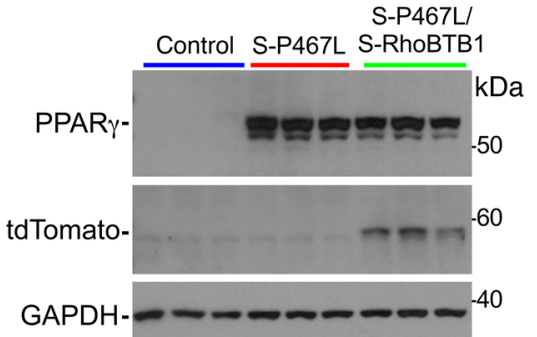<smiles>[Li]</smiles>
Triple
D
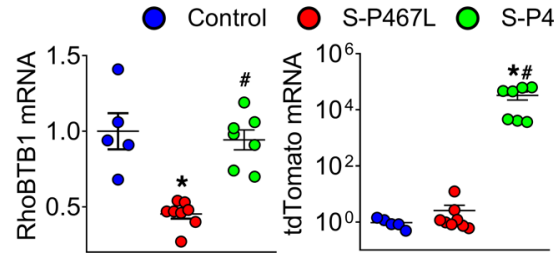

S-P467L/S-RhoBTB1 (S-RhoBTB1)
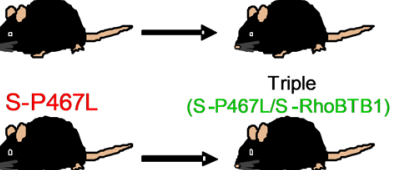

$\mathbf{F}$

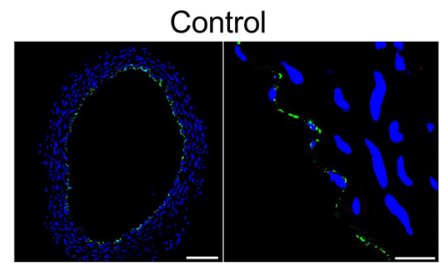

S-P467L/S-RhoBTB1

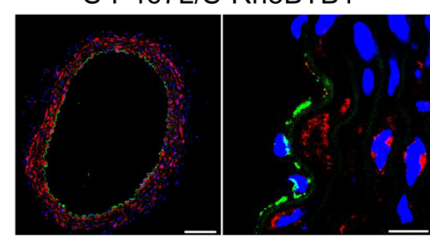

Figure 1. Inducible RhoBTB1 experimental model. (A) Schematic of the inducible RhoBTB1 transgene construct illustrating removal of the loxP-STOP-loxP with consequent expression of RhoBTB1 and tdTomato in response to Cre recombinase. (B) Western blot detecting RhoBTB1 or Myc-tagged RhoBTB1, tdTomato, and GAPDH in HEK293 cells transfected with Myc-RhoBTB1 or RhoBTB1 ${ }^{1 N D}$, with or without a Cre recombinase expression vector. Actual size markers transferred from the blots are shown. (C) Schematic of breeding to generate triple-transgenic mice expressing dominant-negative PPAR $\gamma$ (S-P467L) in vascular smooth muscle along with inducible smooth muscle-specific expression of RhoBTB1 (S-RhoBTB1). (D) Relative mRNA expression of RhoBTB1, tdTomato, and human PPAR $\gamma$ (hPPAR $\gamma$ ) was determined by qPCR in aorta from mice of the indicated strains 3-4 weeks after injection of Tx. Data were normalized to the average control value, set to 1.0. All data represent the mean $\pm \mathrm{SEM} .{ }^{*} P<0.05$ versus control; ${ }^{*} P<0.05$, S-P467L versus S-P467L/S-RhoBTB1 mice; 1-way ANOVA. (E) Western blot of total aortic protein from the indicated mouse strains (treated with TX) probed for PPAR $\gamma$, tdTomato, and GAPDH. Actual size markers transferred from the blots are shown. Shown are 3 representative blots from 7 total samples analyzed for each genotype. (F) Immunostaining of aorta from control and S-P467L/S-RhoBTB1 mice. Red indicates tdTomato and green vWF, a marker of endothelium. DAPI staining (blue) labels nuclei. Scale bars: $100 \mu \mathrm{m}$ (left panels) and approximately $15 \mu \mathrm{m}$ (right panels).

to GPCR agonists (23). Further highlighting the importance of this pathway are genetic data showing that mutations in Cullin-3 cause human hypertension (29). Smooth muscle-specific expression of the hypertension-causing mutation in Cullin-3 causes vascular dysfunction and hypertension through a RhoA and Rho kinase-dependent mechanism (30). However, Cullin-3 mediates its effects on RhoA through the BTB domain-containing protein BACURD, not RhoBTB1 (27). Thus, the mechanism by which decreased expression of RhoBTB1 regulates vasomotor function and $\mathrm{BP}$ remains unclear.

Here, we used a genetic complementation approach to assess the importance of RhoBTB1 and define the mechanism of action of RhoBTB1 in vascular SMCs. The premise is that if the adverse cardiovascular effects of dominant negative PPAR $\gamma$ are mediated by a loss of RhoBTB1 expression, restoring its expression should complement those physiological defects. We have found evidence that RhoBTB1 provides protection from vascular dysfunction characterized by NO resistance, arterial stiffness, and hyperten- sion by restraining the activity of phosphodiesterase 5 (PDE5) and promoting the cyclic $3^{\prime}, 5^{\prime}$-monophosphate-dependent (cGMPdependent) relaxation of vascular SMCs. We also show that this protective effect is not limited to a model of PPAR deficiency but also affects angiotensin-II-induced (Ang-II-induced) hypertension. We identify RhoBTB1 as a gene that provides protection from hypertension, vascular dysfunction, and arterial stiffness.

\section{Results}

We generated transgenic mice (RhoBTB1 ${ }^{\mathrm{IND}}$ ) carrying a conditionally activatable transgene designed for PPAR $\gamma$-independent inducible coexpression of RhoBTB1 and the tdTomato reporter gene in response to Cre recombinase (Figure 1A). Cotransfection of HEK293 cells with vectors encoding RhoBTB1 ${ }^{\mathrm{IND}}$ and Cre recombinase resulted in coexpression of both RhoBTB1 and tdTomato (Figure 1B). See complete unedited blots in the supplemental material. RhoBTB1 ${ }^{\text {IND }}$ mice were bred with SMC-specific tamoxifen-inducible (Tx-inducible) Cre recombinase (SMC-CreER ${ }^{\mathrm{T} 2}$ ) mice to estab- 

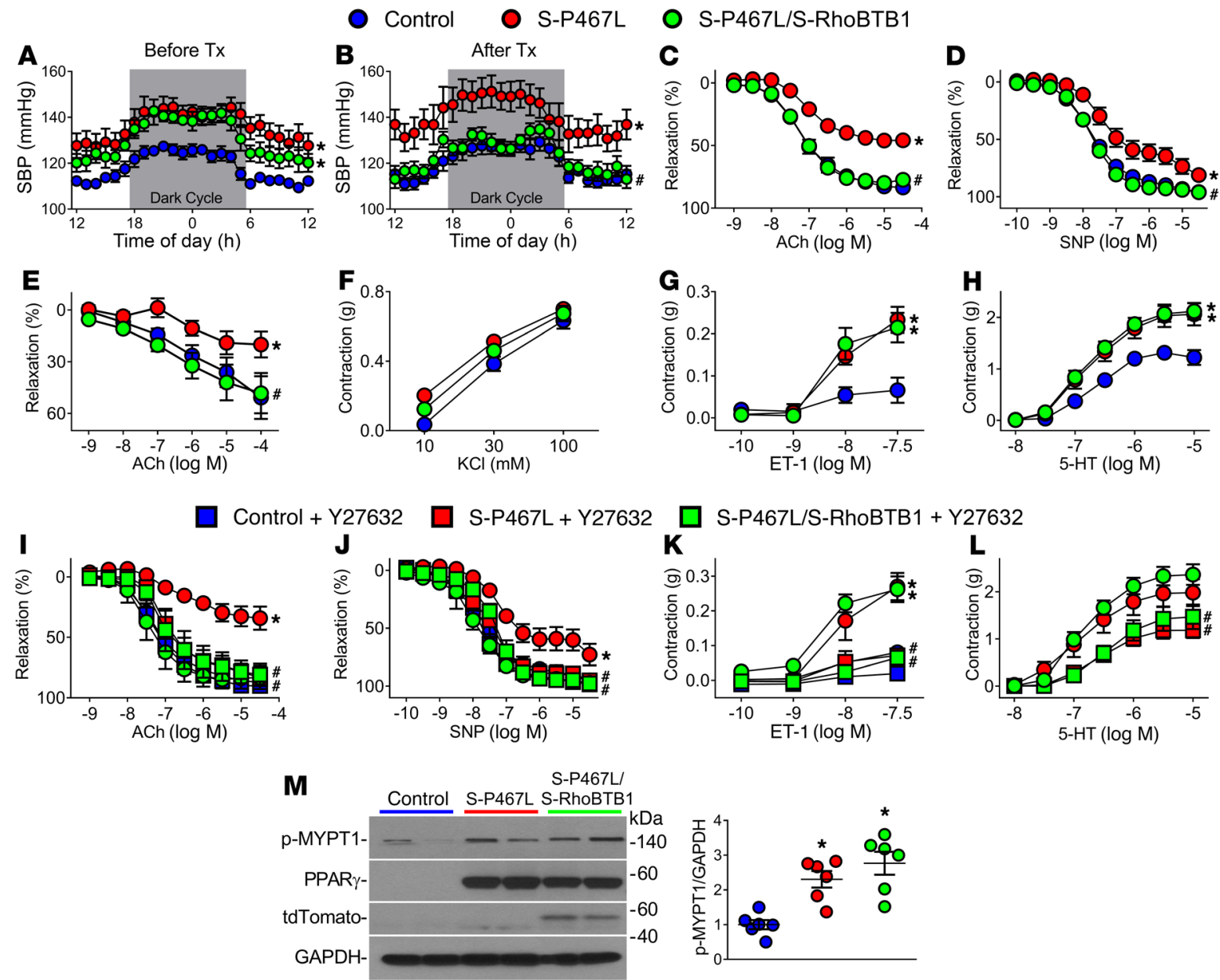

Figure 2. BP and vascular function. (A and B) Systolic BP was measured by radiotelemetry for 1 week in control $(n=8), S-P 467 \mathrm{~L}(n=10)$, and S-P467L/ S-RhoBTB1 mice $(n=8)$ before (A) or 3 to 4 weeks after Tx treatment (B). (C-E) Vascular relaxation in control, S-P467L, and S-P467L/S-RhoBTB1 mice after Tx treatment. Cumulative concentration-response curves for ACh $(n=7-9)$, or SNP $(n=7-9)$ in aorta (C and $\mathbf{D})$ and basilar artery $(n=4-6)(\mathbf{E})$. (F-H) Cumulative concentration-response curves for KCI $(n=8-9)(\mathbf{F})$, ET-1 $(n=4-6)(\mathbf{G})$, and 5-HT $(n=4-6)(\mathbf{H})$ in aorta from Tx-treated mice. (I-L) Cumulative concentration-response curves for ACh $(n=4-5)(\mathrm{I})$, SNP $(n=5-7)(\mathrm{J})$, ET-1 $(n=4-5)(\mathrm{K})$, and 5-HT $(n=3)(\mathrm{L})$ in Y-27632-pretreated aorta from the indicated Tx-treated mice. (M) Western blot detected p-MYPT, PPAR $\gamma$, tdTomato, and GAPDH in total aortic protein from the indicated mice after Tx treatment. Shown are 2 representative blots from 6 total samples analyzed for each genotype. Quantification of the p-MYPT results is shown. Data were normalized to the average control value, set to 1.0. All data represent the mean \pm SEM. ${ }^{*} P<0.05$ versus control; ${ }^{*} P<0.05$, S-P467L versus S-P467L/S-RhoBTB1 mice; 2-way repeated-measures ANOVA.

lish S-RhoBTB1 double-transgenic mice that inducibly expressed RhoBTB1 selectively in vascular smooth muscle (Figure 1C). As a second step, S-RhoBTB1 mice were crossed with S-P467L mice to produce a triple-transgenic experimental model termed S-P467L/ S-RhoBTB1. In this mouse model, reduced expression of endogenous RhoBTB1 in SMCs caused by dominant-negative PPAR $\gamma$ could be reversed by Tx by induction of the PPAR $\gamma$-independent transgene. This genetic complementation strategy was successful, as RhoBTB1 mRNA expression was decreased in S-P467L aorta but was restored to normal levels in Tx-treated S-P467L/S-RhoBTB1 mice (Figure 1D). Tx also induced tdTomato mRNA and protein but, importantly, did not alter P467L PPAR $\gamma$ mRNA or protein levels (Figure 1, D and E). Cre recombinase-mediated expression of tdTomato was specifically localized to SMCs but not endothelium, demonstrating cell specificity (Figure 1F).

To test the hypothesis that loss of RhoBTB1 expression is mechanistically linked to hypertension in S-P467L mice, we mea- sured the effect of restoring RhoBTB1 expression on arterial BP. Both S-P467L and S-P467L/S-RhoBTB1 mice showed isolated systolic hypertension prior to Tx injection (Figure 2A and Supplemental Figure 1; supplemental material available online with this article; https://doi.org/10.1172/JCI123462DS1). Tx treatment had no effect on the BP of S-P467L or control mice (Figure 2B). However, BP in S-P467L/S-RhoBTB1 mice, which was elevated before Tx, returned to normal 2 weeks after Tx treatment, suggesting that restored expression of a single PPAR $\gamma$ target gene, RhoBTB1, reversed the hypertension caused by PPAR $\gamma$ dysfunction. Thoracic aortae from S-P467L mice had impaired vasodilation in response to both acetylcholine (ACh) and sodium nitroprusside (SNP), indicative of NO resistance (Figure 2, C and D). The response to both was corrected after induction of RhoBTB1 expression. We observed that vasodilation was similarly impaired in the basilar artery, a cerebral resistance vessel from S-P467L mice, and was corrected after induction of RhoBTB1 (Figure 2E). 
- Control o S-P467L o S-P467L/S-RhoBTB1

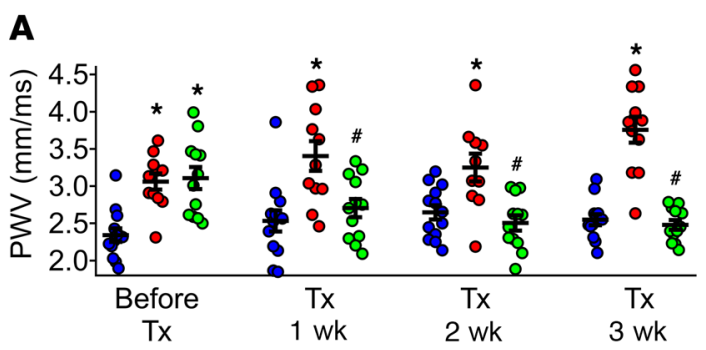

B

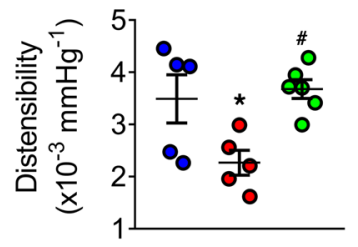

C

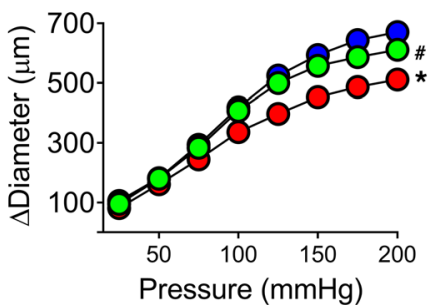

D

E

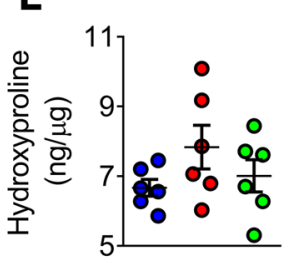

$\mathbf{F}$
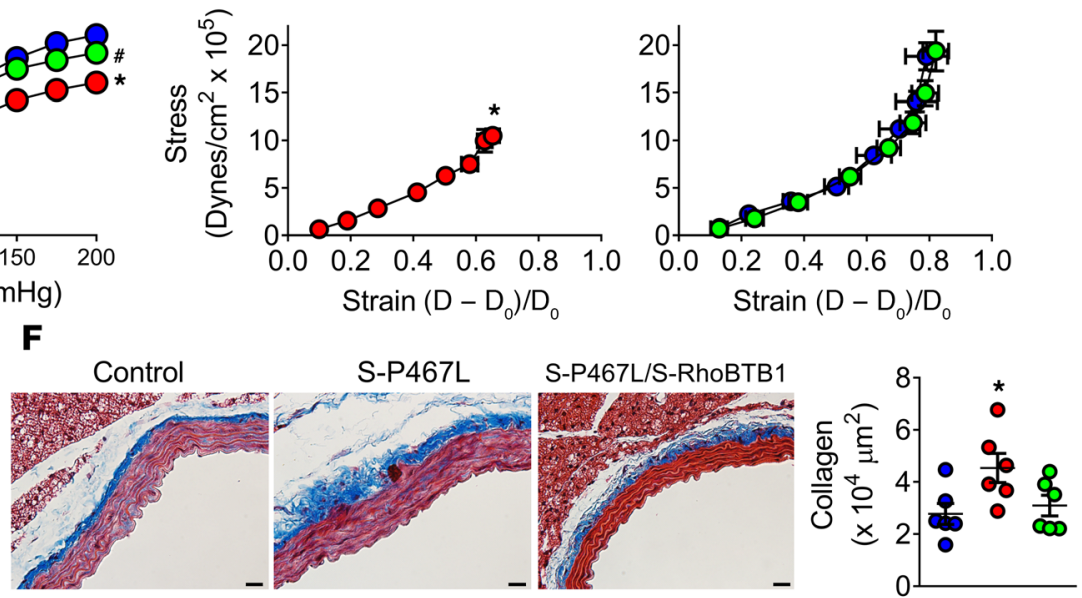

Figure 3. Aortic stiffness. (A) Aortic PWV was measured in control, S-P467L, and S-P467L/S-RhoBTB1 mice before Tx treatment or 1, 2, and 3 weeks after completion of the Tx treatment. (B) Distensibility was calculated at 3 weeks as described in the Methods. (C and D) Compliance curves (C) and stress-strain relationships (D) were constructed from the inner and outer diameters of aortae from these mice 3-4 weeks after Tx treatment. These parameters were measured at $25-\mathrm{mmHg}$ step changes in pressure from 0 to $200 \mathrm{mmHg} . n=5$ for all samples. D, diameter; $D_{0}$, diameter at $0 \mathrm{mmHg}$. (E) Measurement of hydroxyproline as an index of collagen in aorta. (F) Perfusion-fixed sections of thoracic aorta 3-4 weeks after Tx treatment were sectioned and stained with Masson's trichome to stain collagen. Scale bars: $100 \mu \mathrm{m}$. The adventitial collagen area was quantified by planimetry. All data represent the mean \pm SEM. ${ }^{*} P<0.05$ versus control; ${ }^{\#} P<0.05$ S-P467L versus S-P467L/S-RhoBTB1 mice, by 1-way ANOVA or 1-way repeated-measures ANOVA.

Although $\mathrm{KCl}$ contraction was not different between genotypes (Figure 2F), endothelin-1-induced (ET-1-induced) and serotonin-induced (5-HT-induced) contraction was enhanced in S-P467L aorta (Figure 2, G and H). Interestingly, the enhanced contractile responses to 5 -HT and ET-1 were preserved in Tx-treated S-P467L/S-RhoBTB1 mice. Thus, unlike the corrective effects on vasodilation, restoration of RhoBTB1 failed to correct the increased contraction in aortae from S-P467L mice. Inhibition of RhoA and ROCK activity decreases BP and improves vascular dysfunction in S-P467L mice (23). Therefore, we sought to determine whether a RhoBTB1-mediated reduction in RhoA/ROCK signaling might explain the improved vascular function in Tx-treated S-P467L/S-RhoBTB1 mice. Y27632, a selective ROCK inhibitor, reversed the impairment of ACh-induced (Figure 2I) and SNPinduced (Figure 2J) relaxation in S-P467L mice. We detected no change in ACh- or SNP-induced responses in Tx-treated S-P467L/ S-RhoBTB1 mice, as RhoBTB1 induction already corrected both. Y27632 ameliorated the hypercontraction induced by ET-1 and 5-HT in both Tx-treated S-P467L mice and S-P467L/S-RhoBTB1 mice, suggesting a preservation of increased RhoA/ROCK signaling after restoration of RhoBTB1 (Figure 2, K and L). The fact that phosphorylation at Thr696 in the myosin light-chain phosphatase subunit MYPT1 was increased in both S-P467L and S-P467L/
S-RhoBTB1 mice further supports this conclusion (Figure 2M). This suggests that the protective effect of RhoBTB1 expression was not due to inhibition of RhoA/ROCK pathway activity and that PPAR $\gamma$ independently and separately controls vasoconstriction via RhoA/ROCK signaling and vasodilation through RhoBTB1.

Since tdTomato is concurrently expressed with RhoBTB1, we performed control experiments to ensure that tdTomato expression in SMCs would not affect vascular function. To accomplish this, we bred SMC-CreER ${ }^{\text {T2 }}$ mice with ROSA ${ }^{\text {tdTomato }}$ mice, which inducibly express tdTomato. Expression of tdTomato did not alter relaxation in response to either ACh or SNP (Supplemental Figure 2A). We also confirmed that Tx injection on its own did not improve ACh or SNP response curves for S-P467L mice (Supplemental Figure 2B).

$\mathrm{S}-\mathrm{P} 467 \mathrm{~L}$ mice exhibit isolated systolic hypertension, which is typically associated with arterial stiffness (31). We therefore measured aortic stiffness by pulse wave velocity (PWV). We found that aortic PWV was significantly increased in S-P467L mice and that Tx had no effect on PWV in S-P467L or control mice (Figure 3A). Whereas S-P467L/S-RhoBTB1 mice had increased PWV before $\mathrm{Tx}$, this decreased 1 week after Tx treatment and was normalized by 2 weeks. Consistent with the PWV measurements, distensibility was decreased in S-P467L mice and restored in S-P467L/ 
A

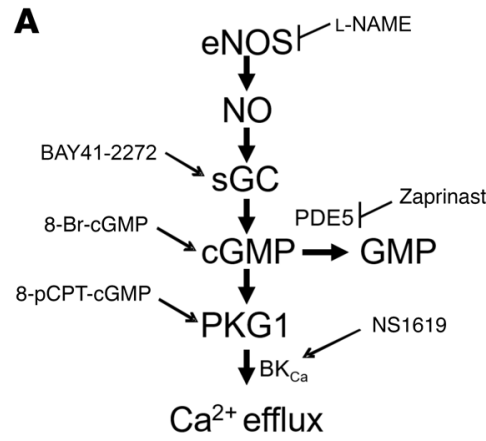

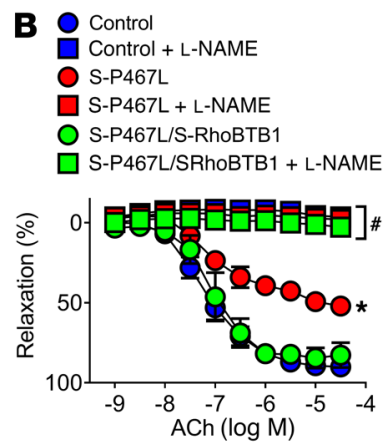

D

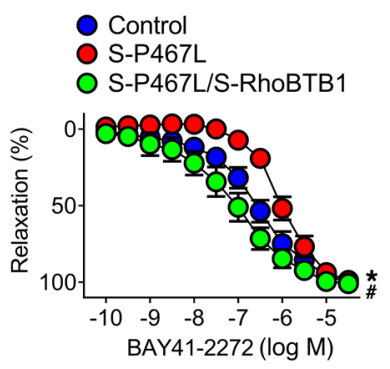

E O Control

- Control, SNP $\left(10^{-6} \mathrm{M}\right)$

- S-P467L

S-P467L, SNP

O S-P467L/S-RhoBTB1

S-P467L/S-RhoBTB1, SNP

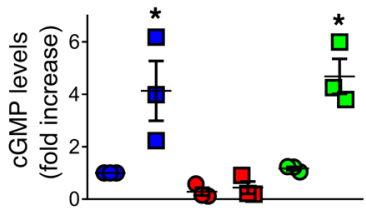

C

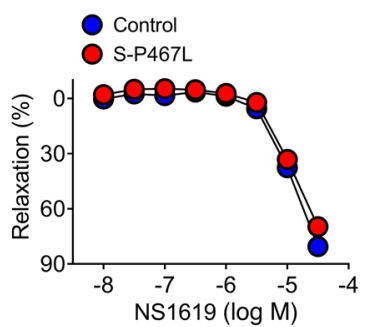

\section{घ0 Control घ0 S-P467L}

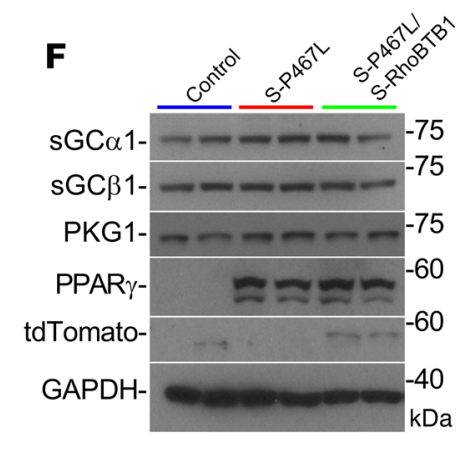

S-P467L/S-RhoBTB1
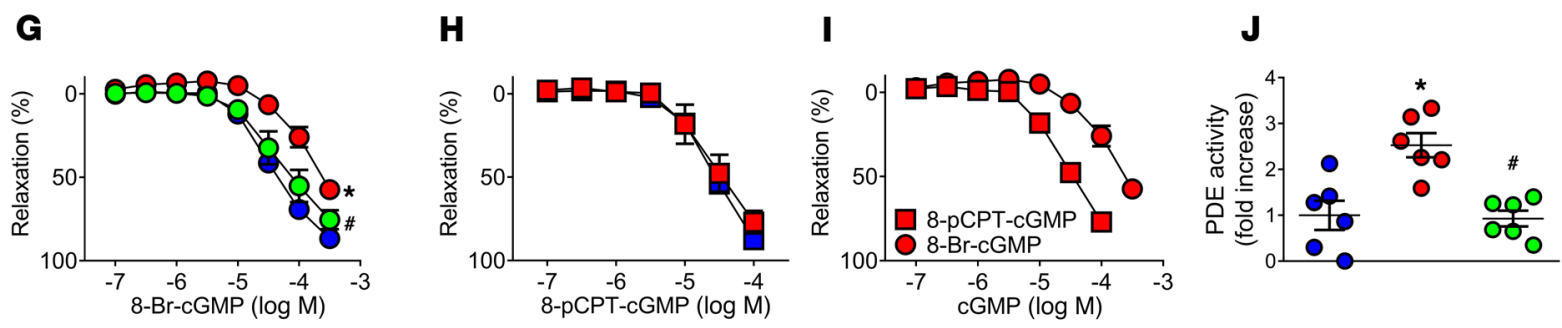

Figure 4. Analysis of the CGMP pathway. (A) Schematic diagram of the NO vasodilation pathway. (B) Aortae from control, S-P467L, or S-P467L/ S-RhoBTB1 mice 3-4 weeks after completion of Tx treatment were treated with or without L-NAME (100 $\mu$ M, 30 min), followed by isometric tension experiments with ACh. $n=4-6$ samples. (C and D) Isometric tension experiments were performed with NS1619 ( $n=4-5)(\mathbf{C})$, or BAY41-2272 ( $n=6-8)$ (D). (E) Production of cGMP in aortae from Tx-treated control, S-P467L, or S-P467L/S-RhoBTB1 mice. Vessel samples were incubated with or without SNP for 20 minutes, and cGMP levels were measured. (F) Western blot of total aortic protein was probed with antisera for the indicated proteins. Shown are 2 representative blots from 4 samples assayed for each genotype. Size markers transferred from the blots are shown. (G and $\mathbf{H})$ Isometric tension experiments were performed with 8-Br-cGMP $(n=7)(\mathbf{G})$ or 8-pCPT-cGMP $(n=4)(\mathbf{H})$ in aortae from control, S-P467L, or S-P467L/S-RhoBTB1 mice 3-4 weeks after Tx treatment. (I) Comparison of vasodilation response to 8-Br-cGMP and 8-pCPT-cGMP. (J) PDE activity in aortae from Tx-treated control, S-P467L, or S-P467L/S-RhoBTB1 mice. All data represent the mean \pm SEM. ${ }^{*} P<0.05$ versus control; ${ }^{*} P<0.05$, S-P467L versus S-P467L/S-RhoBTB1 mice; 1 -way ANOVA or 2-way repeated-measures ANOVA.

S-RhoBTB1 mice (Figure 3B). The pressure-diameter relationship and stress-strain curve were impaired in aorta from S-P467L mice (Figure 3, C and D). These effects were reversed in S-P467L/ S-RhoBTB1 mice 3 weeks after Tx. The level of hydroxyproline, an index of collagen, trended to increase in aorta from S-P467L mice compared with that from control mice and decreased in aorta from S-P467L/S-RhoBTB1 mice (Figure 3E). The increase in collagen was confirmed by Masson's trichrome staining, which revealed that adventitial collagen was increased in S-P467L mice but not Tx-treated S-P467L/S-RhoBTB1 mice (Figure 3F). These data suggest that restoration of RhoBTB1 expression can rapidly regress arterial stiffness.

Because RhoBTB1 normalized vasodilation but did not ameliorate the enhanced contraction in S-P467L mice, we focused our search for targets of RhoBTB1 function on the NO-dependent vasodilation pathway (Figure 4A). ACh-induced relaxation is dependent on endothelial NO synthase (eNOS) in arteries such as the aorta and the basilar artery. Pretreatment with the NOS inhibitor L-NAME completely abolished ACh-induced relaxation in mice of all genotypes, including S-P467L/S-RhoBTB1 mice (Figure 4B). This suggests that (a) the restoration of vasodilation in S-P467L/ S-RhoBTB1 mice was due to a restoration of the NO-dependent pathway and not some alternative pathway, and (b) the defect in S-P467L mice probably occurs downstream of eNOS. Vasodilation induced by the calcium-activated potassium channel $\left(\mathrm{BK}_{\mathrm{Ca}}\right)$ opener NS1619 was normal in S-P467L (Figure 4C). Although we recognize that there are many channels that participate in the vasodilation pathway, the fact that there was no defect in vasodilation to 
A

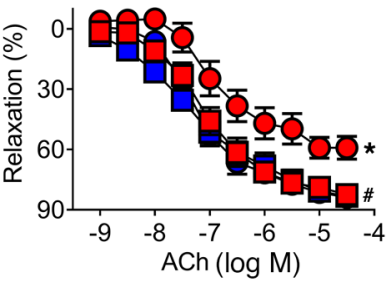

D

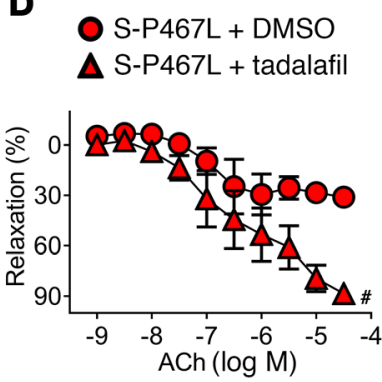

B

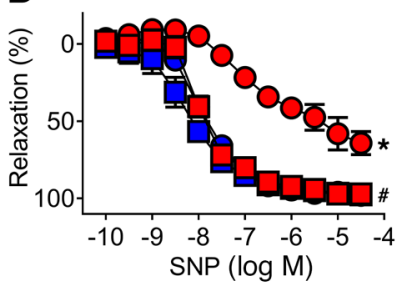

E

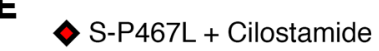
$\nabla$ S-P467L + MBCQ

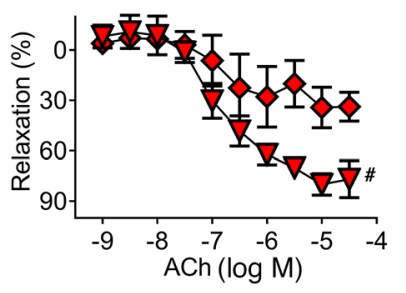

C

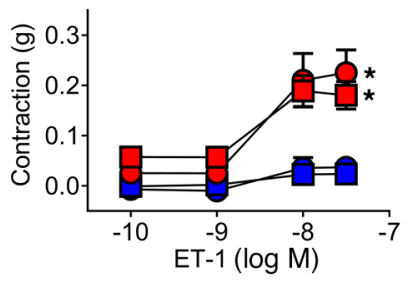

$\mathbf{F}$

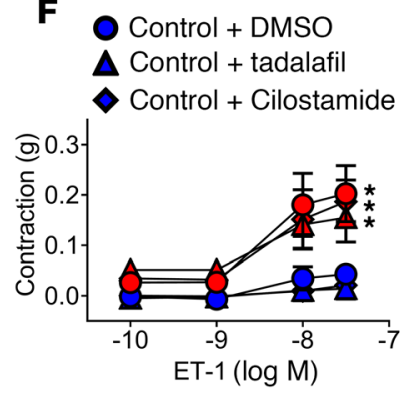

- Control $\boldsymbol{\Delta}$ Control + tadalafil

○ S-P467L

$\Delta S-P 467 L+$ tadalafil
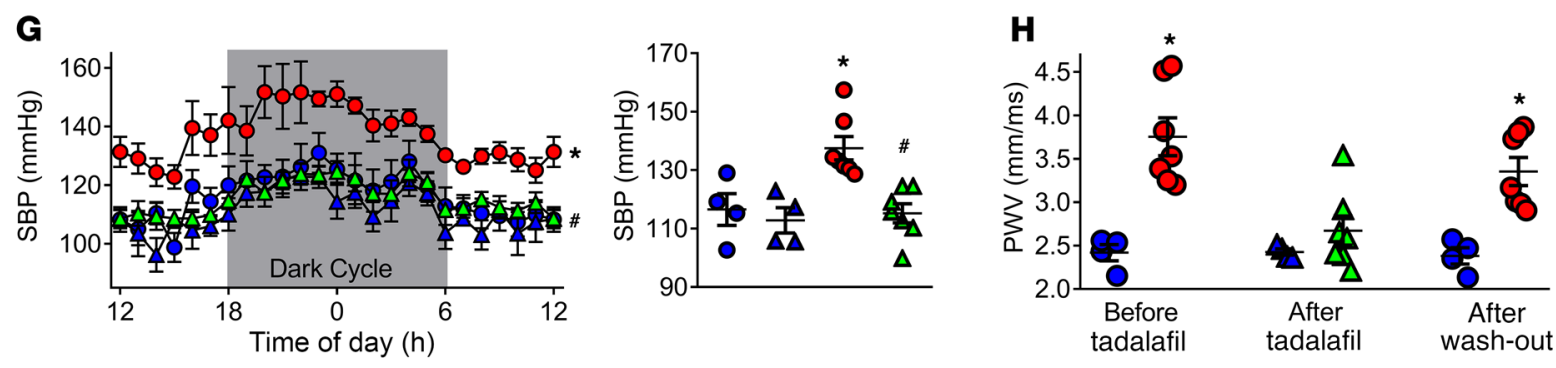

Figure 5. PDE activity and cardiovascular effect of PDE5 inhibition. (A-C) Concentration-dependent curves in response to ACh $(n=6-7)(\mathbf{A})$, SNP $(n=4)$ (B), or ET-1 $(n=4-5)$ (C) in control or S-P467L aortae pretreated or not with Zaprinast (5 $\mu$ M). (D and E) Concentration-dependent relaxation in response to ACh in aorta from S-P467L mice treated with the indicated PDE inhibitor (100 nM tadalafil, 1 M MBCQ, or $1 \mu$ M Cilostamide). $n=4$ samples for each. (F) Concentration-dependent contraction in response to ET-1 in aorta from S-P467L or control mice treated with the indicated PDE inhibitor (100 nM tadalafil or $1 \mu$ M Cilostamide). $n=4$ samples for each. ${ }^{*} P<0.05$ versus control; ${ }^{\#} P<0.05$ versus S-P467L (vehicle); 2 -way repeated-measures ANOVA (A-F). ${ }^{\#} P<$ 0.05 , S-P467L plus cilostamide versus S-P467L plus MBCQ; 2-way repeated-measures ANOVA (E). (C) Systolic BP was measured by radiotelemetry in control $(n=4)$ and S-P467L $(n=7)$ mice before and after tadalafil treatment (10 mg/kg, $5 \mathrm{~d})$. Data are plotted as an hourly (left) and 24-hour (right) average. (H) Aortic PWV was measured in control and S-P467L mice before and 1 week after tadalafil treatment and then 2 weeks after terminating the treatment (after washout). All data represent the mean \pm SEM. ${ }^{*} P<0.05$ versus control; ${ }^{*} P<0.05$, tadalafil versus vehicle; $t$ test (H) or 2 -way ANOVA (A-G).

the $\mathrm{BK}_{\mathrm{Ca}}$ channel opener suggests that the defect in this pathway is most likely upstream of calcium channel activity. Since cGMPdependent activation of protein kinase G (PKG) is an important regulator of smooth muscle tone, we focused on the cGMP pathway as a target of PPAR $\gamma /$ RhoBTB1 regulation. Relaxation in response to the soluble guanylate cyclase (sGC) stimulator BAY412272 was impaired in S-P467L mice and was improved in Tx-treated S-P467L/S-RhoBTB1 mice (Figure 4D). Similarly, we found that the SNP-induced increase in cGMP was markedly impaired in aortae from S-P467L and corrected in S-P467L/S-RhoBTB1 aortae (Figure 4E). Although, these data suggest a potential defect in cGMP production, we detected no difference between genotypes in the levels of the $\alpha 1$ and $\beta 1$ subunits of sGC (Figure 4F). Similarly, protein and mRNA expression of PKG1, the downstream target of cGMP, was not altered in S-P467L mice (Figure 4F and Supplemental Figure 3A). PKG2 mRNA was barely detectable in SMCs (Ct levels = 34.5), but PKG2 mRNA was elevated in aortae from both S-P467L and S-P467L/S-RhoBTB1 mice (Supplemental Fig- ure 3B). These data led us to consider that the defect in the cGMP response may reflect differences in cGMP stability rather than synthesis. Consistent with this observation, aorta from S-P467L mice showed impaired relaxation in response to 8-Bromo-cGMP (8-Br-cGMP) (Figure 4G), which was reversed by restoration of RhoBTB1 expression in SMCs. In contrast, aorta from S-P467L mice showed normal relaxation in response to 8-(4-chlorophenylthio)-cGMP (8-pCPT-cGMP) (Figure 4H). A direct comparison of vasodilation in response to the $2 \mathrm{cGMP}$ analogs showed nearly a $\log$ difference in the dose required to maximally dilate the aortae from S-P467L mice (Figure 4I). Notably, 8-pCPT-cGMP is more resistant to PDE-mediated hydrolysis, whereas 8-Br-cGMP is sensitive. That total PDE activity was increased in aorta from S-P467L mice and was reduced to normal levels in aorta from S-P467L/ S-RhoBTB1 mice provides further evidence for a defect in cGMP stability (Figure 4J). These results strongly suggest that the defect in S-P467L resulted from increased PDE-mediated hydrolysis of cGMP, which was rescued by RhoBTB1 expression. 
A

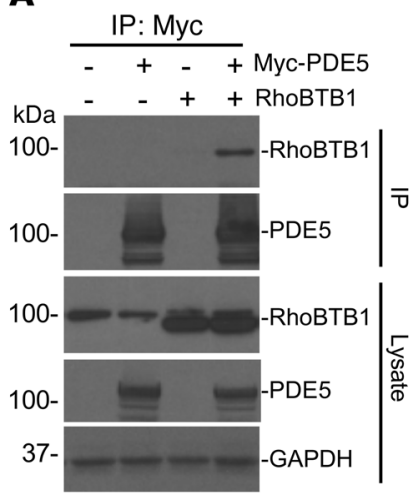

D

- Control, $0 \mathrm{~h}$

- Control, $16 \mathrm{~h}$ DMSO

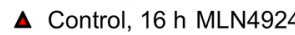

a Control, $72 \mathrm{~h}$ DMSO

a Control, 72 h MLN4924

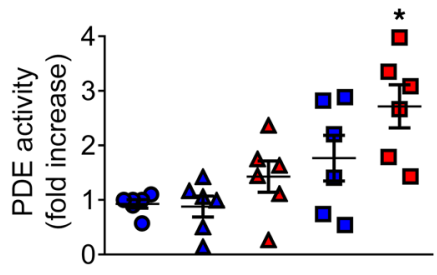

B

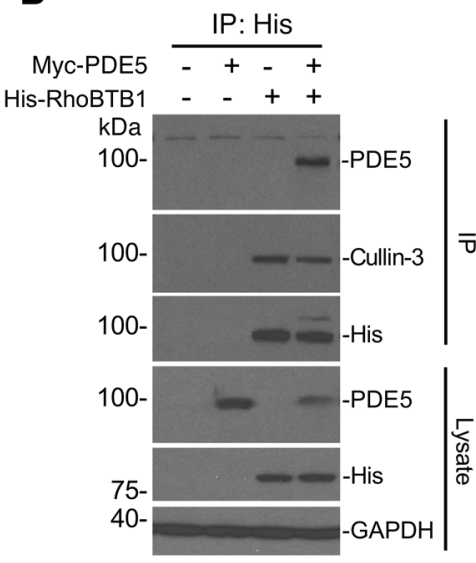

E

- Control, $\mathrm{Oh}$

A Control, 72 h MLN4924

$\Delta$ Control, 72 h MLN4924 + tadalafil

a Control, $72 \mathrm{~h}$ tadalafil

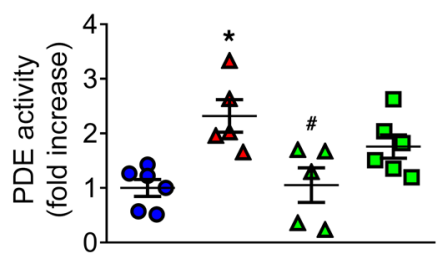

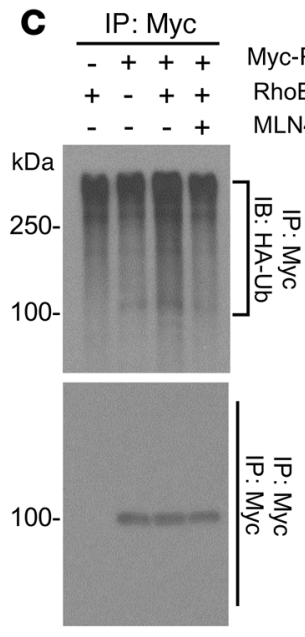

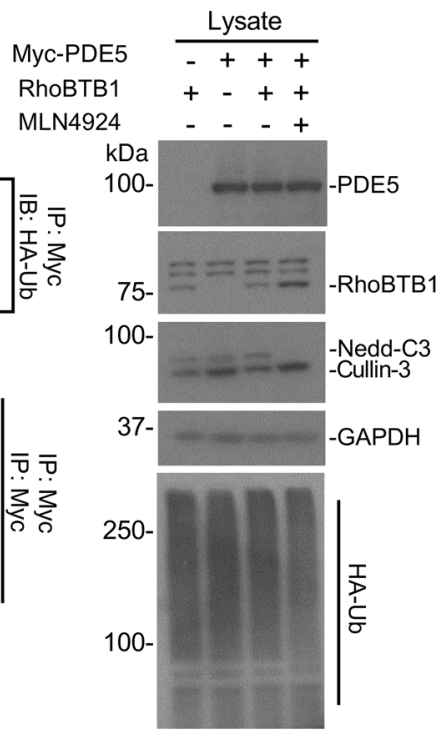

$\mathbf{F}$

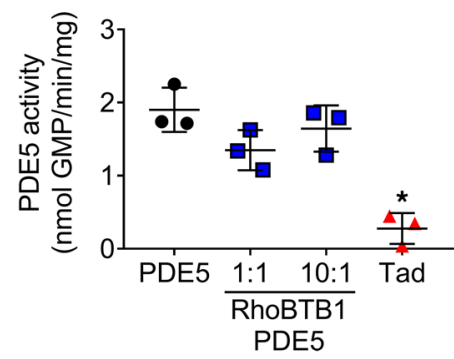

Figure 6. Molecular mechanism of RhoBTB1 on PDE5. (A and B) Reciprocal co-IP of PDE5 and RhoBTB1 in HEK293 cells transfected with Myc-tagged PDE5 and untagged RhoBTB1 (A) or His-tagged RhoBTB1 (B). Cells in B were treated with MLN4924 (1 $\mu$ M) for 16 hours. Western blots were probed with the indicated antisera. IP and lysates are labeled. Molecular weight markers were transferred from the original blots. (C) Ubiquitination of PDE5 in HEK293 cells transfected with vectors expressing HA-tagged ubiquitin (Ha-Ub) and Cullin-3 in all lanes and, as indicated, Myc-tagged PDE5 and RhoBTB1. Cells were treated with MC132 for the last 2 hours, prior to lysis. The indicated sample was pretreated with MLN4924 (1 $\mu$ M) for 16 hours. MLN4924 prevents neddylation of Cullin-3 (note the loss of Nedd-C3, the neddylated form of Cullin-3). IP was performed under stringent denaturing conditions to ensure detection of only ubiquitinated PDE5. Under these conditions, RhoBTB1 was not pulled down by PDE5. Western blots were probed with the indicated antisera. IP and lysates are labeled. Molecular weight markers were transferred from the original blots. (D) PDE activity in aorta from control mice pretreated with either vehicle (DMSO) or MLN4924 (1 $\mu \mathrm{M})$ for the indicated durations. (E) PDE activity in aorta from control mice pretreated with either MLN4924 (1 $\mu$ M) or tadalafil $(1 \mu \mathrm{M})$ for 72 hours. ${ }^{*} P<0.05$ versus control; ${ }^{\#} P<0.05$, MLN4924 plus tadalafil versus MLN4924; 1-way ANOVA. (F) PDE activity in vitro using purified RhoBTB1 and PDE5 or tadalafil $(2 \mu \mathrm{M}) .{ }^{*} P<0.05$ versus PDE5 alone by 1 -way ANOVA. All data represent the mean \pm SEM.

PDE5 is the major cGMP-hydrolyzing PDE that controls smooth muscle relaxation (32). PDE5 mRNA was robustly expressed in SMCs, but its level of expression was not changed in S-P467L or S-P467L/S-RhoBTB1 mice (Supplemental Figure 4). PDE6, PDE9, and PDE11 were very poorly expressed in the aorta and also did not change among genotypes (Supplemental Figure 4). Though it is known that PDE1 and PDE3 also are expressed in smooth muscle, we observed no difference in expression of PDE1a, PDE1c, or PDE3b between S-P467L and S-P467L/ S-RhoBTB1 mice (Supplemental Figure 4).

To examine the effect of PDE activity on vascular dysfunction in S-P467L mice, zaprinast, an inhibitor of several PDEs including PDE5 (also PDE6, PDE9, and PDE11), was initially used. Zaprinast pretreatment completely reversed the impairment of AChinduced (Figure 5A) or SNP-induced (Figure 5B) relaxation in aortae from S-P467L mice. Consistent with contraction being reg- ulated independently of RhoBTB1, Zaprinast did not correct the enhancement of ET-1 contraction in S-P467L mice (Figure 5C). To confirm that the action of Zaprinast was due to inhibition of PDE5, we repeated the experiments using the PDE-specific inhibitors tadalafil or MBCQ for PDE5 and cilostamide for PDE3. Pretreatment with tadalafil or MBCQ corrected ACh-mediated relaxation in S-P467L mice (Figure 5, D and E). In contrast, the PDE3 inhibitor Cilostamide could not restore relaxation in response to ACh in S-P467L mice. None of the PDE inhibitors tested had any effect on vasodilation of the aortae from control mice (Supplemental Figure 5). As with Zaprinast, we observed no improvement in ET-1-mediated contraction after pretreatment with tadalafil or Cilostamide (Figure 5F). These results suggest that the impairment of vasodilation in S-P467L mice was caused by increased PDE5 activity, which was reversed by RhoBTB1. To determine whether PDE5 activity is involved in hypertension and aortic stiffness caused by 
A
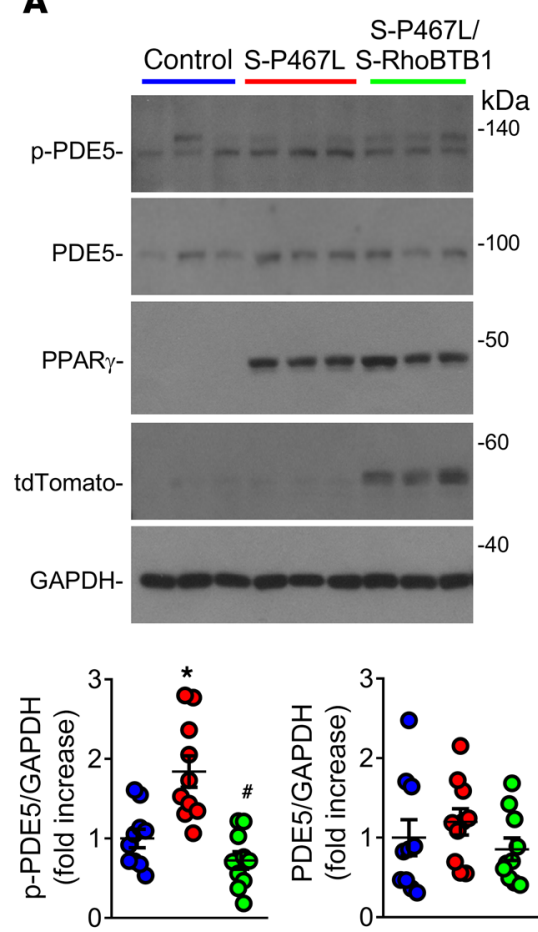

B

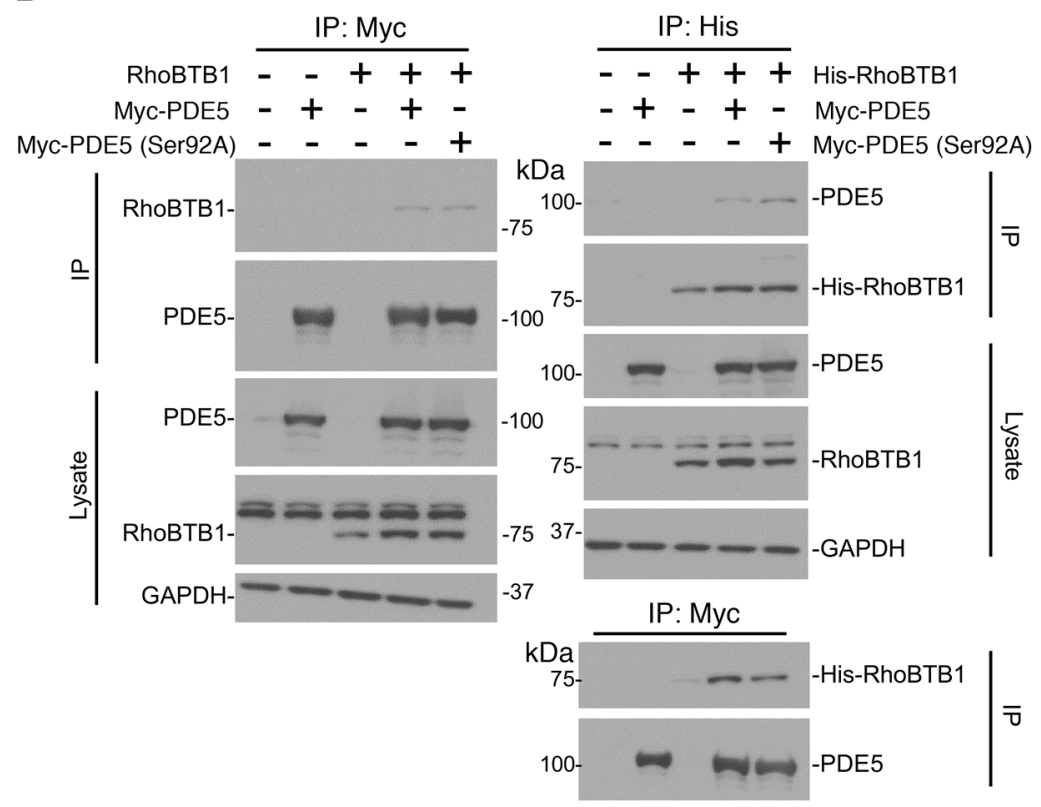

Figure 7. Role of p-PDE5. (A) Western blot of total aortic protein from Tx-treated mice detecting p-PDE5 and total PDE5, PPAR $\gamma$, tdTomato, and GAPDH Size markers transferred from the original blots are shown. Shown are 3 representative blots from 10 samples assayed. The levels of $p-P D E 5$ and total PDE5 from all 10 samples were quantified. Data were normalized to the average control value, set to $1.0 .{ }^{*} P<0.05$ versus control; ${ }^{\#} P<0.05$ versus S-P467L; 1-way ANOVA. (B) Reciprocal co-IP of WT and Ser92A mutation in PDE5 and RhoBTB1 in HEK293 cells transfected with either Myc-tagged PDE5 or Myc-tagged PDE5 (Ser92A) and either untagged RhoBTB1 or His-tagged RhoBTB1. Cells in B were treated with MLN4924 (1 $\mu$ M) for 16 hours. Western blots were probed with the indicated antisera. IP and lysates are labeled. Molecular weight markers were transferred from the original blots.

dominant-negative PPAR $\gamma$, we administered tadalafil to S-P467L mice. Treatment with tadalafil 3 times per week lowered systolic BP in S-P467L mice to control levels (Figure 5G). Systolic BP was not altered by tadalafil in control mice. Increased PWV in S-P467L mice was also improved by tadalafil treatment (Figure $5 \mathrm{H}$ ). However, 10 days after discontinuing tadalafil treatment, increased PWV, and thus arterial stiffness, returned in S-P467L mice. These data suggest that PDE5 inhibition phenocopies the restoration of RhoBTB1 and provide further evidence that RhoBTB1 mediates its protective effects through PDE5 inhibition. Moreover, the data suggest a direct relationship between cGMP and vascular stiffness.

As a BTB domain-containing protein, RhoBTB1 may act as a substrate adaptor for Cullin-3. We hypothesized that just as BACURD acts as a Cullin-3 adaptor for RhoA, RhoBTB1 acts as a Cullin-3 adaptor for PDE5. We performed co-IP experiments in HEK293 cells using Myc-tagged PDE5 and untagged RhoBTB1. IP of PDE5 efficiently coprecipitated RhoBTB1 (Figure 6A). Likewise, we observed that IP of His-tagged RhoBTB1 coprecipitated PDE5 (Figure 6B). It is notable that RhoBTB1 also coimmunoprecipitated Cullin-3, suggesting its incorporation into the CRL3 complex. Interestingly, the IP of PDE5 was more efficient in the presence of MLN4924, an inhibitor of the neddylation of Cullin proteins (data not shown). Neddylation is required for Cullin-dependent ubiquitin ligase activity, and we show that inhibition of neddylation prevents ubiquitination of PDE5 by the CRL3 complex and reduction in PDE activity. First, we observed RhoBTB1-dependent ubiquiti- nation of PDE5 in HEK293 cells overexpressing Cullin-3, which was impaired by inhibiting the Cullin family of $\mathrm{E} 3$ ubiquitin ligases with MLN4924 (Figure 6C). Note that MLN4924 treatment eliminated the neddylated form of Cullin-3 in the lysate. Replication experiments showed RhoBTB1-dependent ubiquitination of PDE5 in HEK293 cells either in the presence of overexpressed Cullin-3 (Supplemental Figure 6A) or only endogenous expression of Cullin-3 (Supplemental Figure 6B). Second, MLN4924 treatment of aorta in culture for 72 hours increased total PDE activity (Figure 6D). This activity is attributed to PDE5, because it was reversed by tadalafil in a replication cohort (Figure 6E). The apparent increase in PDE activity by tadalafil alone was not significant and was similar to incubation with vehicle. Given the association between RhoBTB1 and PDE5, we tested whether RhoBTB1 could inhibit PDE5 activity in vitro using purified proteins. Consistent with a role for an active CRL3 complex, RhoBTB1, even at 10-fold molar excess, was unable to significantly inhibit PDE5 activity (Figure 6F). That tadalafil efficiently inhibited PDE5 activity in vitro demonstrated the fidelity of the assay. These data suggest that RhoBTB1 acts as a substrate adaptor for PDE5, delivering PDE5 to the CRL3 complex for ubiquitination, and that the pathway is operant in vascular smooth muscle.

PDE5 is activated by PKG-mediated phosphorylation at Ser92 (33). Although there was no change in the level of the nonphosphorylated form of PDE5, we detected a significant increase in Ser92 phosphorylated PDE5 (p-PDE5) in aortae from S-P467L 

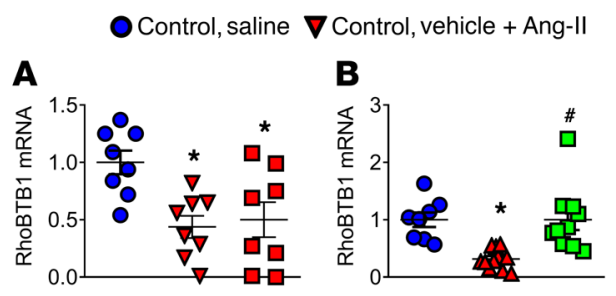

DControl, Tx + Ang-II $\Delta$ S-RhoBTB1, vehicle + Ang-II

口S-RhoBTB1, Tx + Ang-II
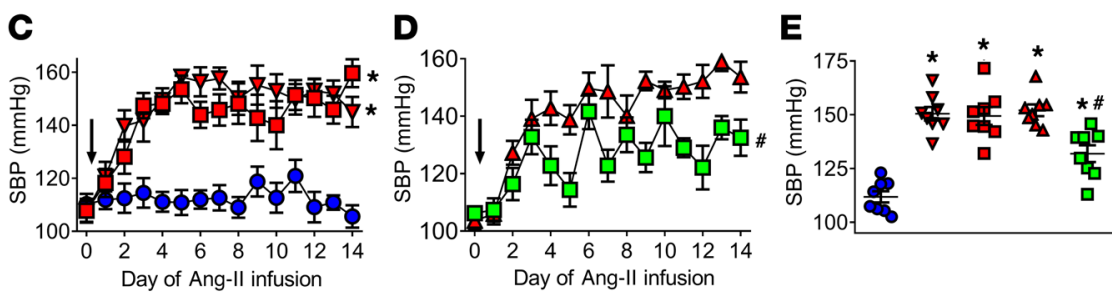

Day of Ang-II infusion
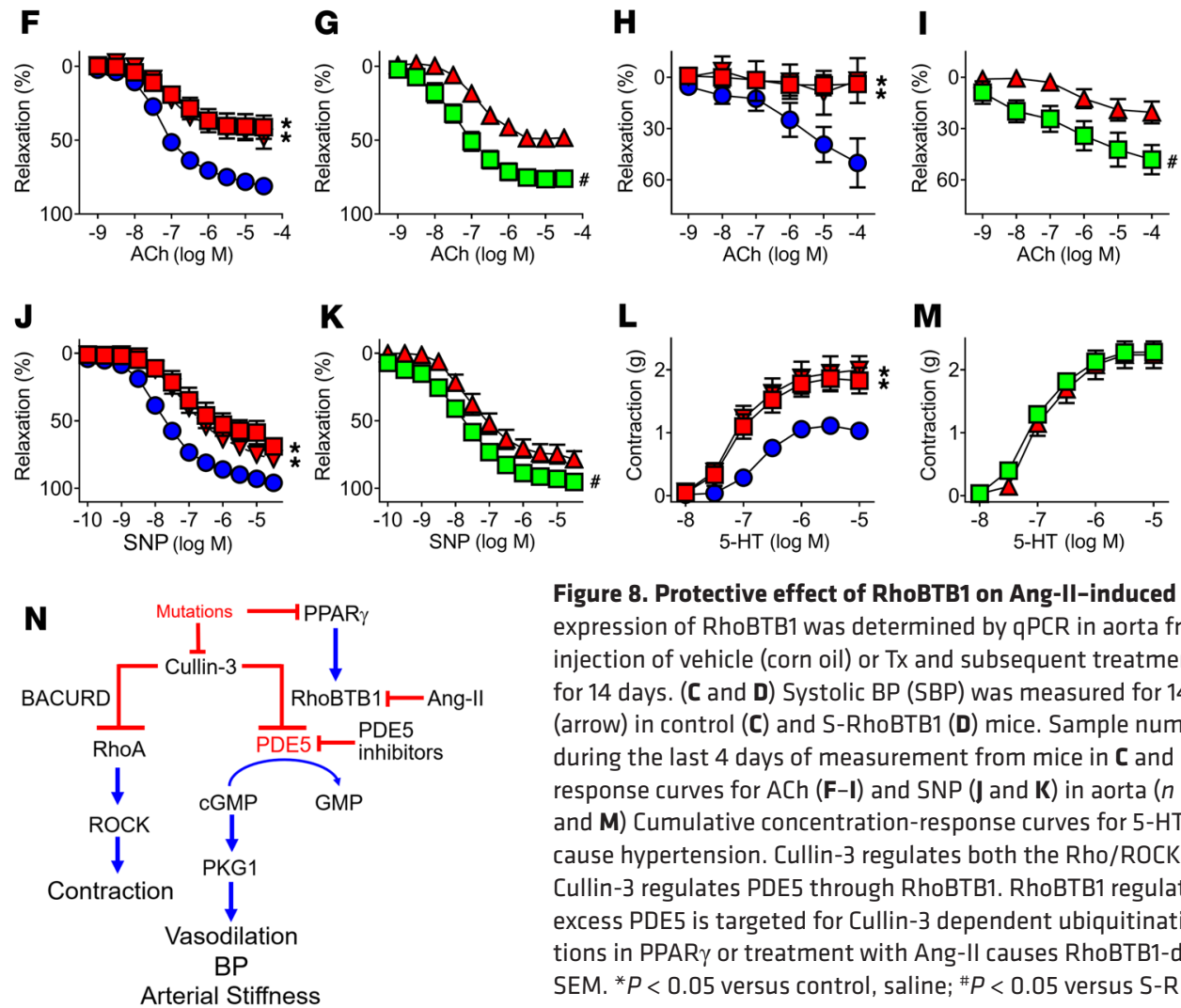

Figure 8. Protective effect of RhoBTB1 on Ang-II-induced hypertension. (A and B) Relative mRNA expression of RhoBTB1 was determined by qPCR in aorta from control (A) or S-RhoBTB1 (B) mice after injection of vehicle (corn oil) or Tx and subsequent treatment with Ang-II (490 ng $/ \mathrm{min} / \mathrm{kg})$ or saline for 14 days. (C and $\mathbf{D})$ Systolic BP (SBP) was measured for 14 days after initiation of Ang-II infusion (arrow) in control (C) and S-RhoBTB1 (D) mice. Sample numbers are indicated in E. (E) Cumulative SBP during the last 4 days of measurement from mice in $\mathbf{C}$ and $\mathbf{D}$. (F-K) Cumulative concentrationresponse curves for ACh $(\mathbf{F}-\mathbf{I})$ and SNP $(\mathbf{J}$ and $\mathbf{K})$ in aorta $(n=5-8)$ and basilar artery $(n=4)(\mathbf{H}-\mathbf{I})$. (L and $\mathbf{M}$ ) Cumulative concentration-response curves for $5-\mathrm{HT}$. (N) Mutations in either Cullin-3 or PPAR $\gamma$ cause hypertension. Cullin-3 regulates both the Rho/ROCK pathway through BACURD, whereas Cullin-3 regulates PDE5 through RhoBTB1. RhoBTB1 regulates the activity of PDE5 by ensuring that excess PDE5 is targeted for Cullin-3 dependent ubiquitination and proteasomal degradation. Mutations in PPAR $\gamma$ or treatment with Ang-II causes RhoBTB1-deficiency. All data represent the mean \pm SEM. ${ }^{*} P<0.05$ versus control, saline; ${ }^{\#} P<0.05$ versus S-RhoBTB1 $V$ plus Ang-II; 1 -way ANOVA or 2-way repeated-measures ANOVA

mice, which was reversed in S-P467L/S-RhoBTB1 mice (Figure 7A). We next considered the possibility that RhoBTB1 may differentially bind to PDE5 and p-PDE5. PKG-dependent PDE5 phosphorylation at Ser92 is a major PDE5 activation pathway in human SMCs (34). HEK293 cells were transfected with native RhoBTB1 or an N-terminal His-tagged RhoBTB1 alone or in the presence of PDE5 or a mutant PDE5 substituting alanine for serine at position 92 (Ser92A). Interestingly, RhoBTB1 and Histagged RhoBTB1 bound equally to both the WT and mutant forms of PDE5 (Figure 7B).

Finally, we asked whether RhoBTB1 can regulate BP and vascular function in another model of hypertension. Interestingly, we found that RhoBTB1 mRNA expression in aortae from C57BL/6 mice was decreased in response to Ang-II infusion (490 ng/min/ $/ \mathrm{kg}$, 2 weeks) and that Tx had no effect on the level of RhoBTB1 mRNA expression in control mice (Figure 8A). To examine the hypothesis that restoring RhoBTB1 might prevent hypertension and vascular dysfunction caused by Ang-II, we studied double-transgenic mice (S-RhoBTB1) generated by breeding SMC-CreER ${ }^{\mathrm{T} 2}$ mice with mice carrying the inducible RhoBTB1 transgene. S-P467L mice were not used in these experiments. We observed that RhoBTB1 expression was decreased in aortae from S-RhoBTB1 mice infused with Ang-II before Tx treatment but was restored to normal levels after Tx treatment (Figure 8B). Restoration of RhoBTB1 did not alter baseline systolic BP in the absence of Ang-II (data not shown), and Tx had no effect on the Ang-IIinduced pressor response in control mice (Figure 8C). However, the Ang-II-induced pressor response was significantly attenuated by RhoBTB1 expression (Figure 8D). Analysis of the composite data revealed a 50\% protection from Ang-II-induced hypertension (Figure 8E). We also observed cardiac hypertrophy in AngII-infused control mice, which was prevented in S-RhoBTB1 mice treated with Tx (Supplemental Figure 7). ACh-induced relaxation of the thoracic aorta was impaired by Ang-II (Figure 8F) and was prevented by restoration of RhoBTB1 (Figure 8G). Similarly, the impairment in ACh-induced relaxation of the basilar artery (Figure $8 \mathrm{H}$ ) was corrected by RhoBTB1 restoration (Figure 8I). We observed a similar improvement for SNP-induced relaxation in aorta (Figure 8, J and K). Consistent with the results in S-P467L mice, Ang-II increased 5-HT-induced contraction in aorta (Figure 
8L), which was preserved after RhoBTB1 restoration (Figure 8M). These results demonstrate that RhoBTB1 protects against Ang-IIinduced defects in relaxation, but not in contraction, similar to its protection against PPAR $\gamma$ dysfunction.

\section{Discussion}

Dominant-negative mutations in PPAR $\gamma$ cause hypertension and insulin resistance (11), which are separable phenotypes. For example, adipose-specific deletion of PPAR $\gamma$ causes insulin resistance (35), whereas mice expressing hypertension-causing mutations in PPAR $\gamma$ specifically in vascular SMCs exhibit hypertension and vascular dysfunction but no evidence of insulin resistance or diabetes (16). Pharmacological studies revealed that the increased BP and vascular dysfunction caused by mutations in PPAR $\gamma$ depends on increased RhoA/ROCK activity, because inhibition of ROCK lowered arterial pressure and restored normal vasodilation (23). Thus, we initially sought a mechanistic link between the transcriptional activity of PPAR $\gamma$ and genes related to the RhoA/ROCK pathway. Transcriptomic analysis of 258 genes whose expression in aorta changed in response to either PPAR $\gamma$ activation or mutations in PPAR $\gamma$ revealed that DOCK11 and RhoBTB1 were the only RhoA/ ROCK-related genes identified $(36,37)$. There is very little published information on DOCK11 except for a report that it may act as a guanine nucleotide-exchange factor that activates Cdc42 (38). We previously showed that there was no difference in activation of Cdc42 in the aortae of S-P467L mice, and thus this effectively ruled out DOCK11 as a viable candidate (23). RhoBTB1 is an atypical Rho GTPase that is larger than other family members and contains both a highly divergent and nonfunctional $\mathrm{N}$-terminal GTPase domain and a BTB domain (24). BTB domain-containing proteins are characterized by their ability to bind to the Cullin-3 E3 ring ubiquitin ligase complex and to act as adaptors that deliver substrates for ubiquitination to the CRL3 complex (25). Like PPAR $\gamma$, mutations in Cullin-3 cause human hypertension, suggesting that this biochemical pathway is important physiologically (29). All of these factors led us to focus on identifying the function of RhoBTB1.

We developed a genetic complementation strategy by inducing reexpression of RhoBTB1 selectively in vascular smooth muscle in S-P467L mice through a PPAR $\gamma$-independent pathway. Our key findings are that restoration of RhoBTB1 expression ameliorated the hypertension and impaired the vasodilation observed in S-P467L mice through a Cullin-3- and PDE5-dependent mechanism. Moreover, Ang-II, the key effector of the renin-angiotensin system, decreased expression of RhoBTB1 mRNA in aorta, and its restoration prevented vascular dysfunction and attenuated hypertension. Thus, RhoBTB1 was cardioprotective in at least 2 different models of hypertension.

We propose that under normal conditions, RhoBTB1 tightly regulates the activity of PDE5 by ensuring that excess PDE5 is targeted for ubiquitination and proteasomal degradation (see model in Figure $8 \mathrm{~N}$ ). Our data suggest that it is unlikely that RhoBTB1 directly inhibits PDE5 through their interaction but that this requires the ubiquitination pathway. This ensures tight control of cGMP levels and the maintenance of a balance of vasoconstrictor and vasodilator tone. Under pathological conditions characterized by mutations in PPAR $\gamma$, which impair RhoBTB1 expression, or mutations in Cullin-3, which impair RhoBTB1 activity, increased
PDE5 protein and activity increased the rate of cGMP hydrolysis. In our study, the cGMP generated in response to NO-mediated GC activity was degraded by PDE5, thereby impairing NOdependent vasodilation. Under physiological conditions, modulation of PPAR $\gamma$ activity may control the level of RhoBTB1 and thus PDE5 and cGMP. Indeed, PPAR $\gamma$ activity itself can be modulated by posttranslational modification such as phosphorylation, SUMOylation, acetylation, S-nitrosylation, $O$-GlcNAcylation, and ubiquitination (39). The Ang-II-induced decrease in RhoBTB1 expression may be because the Ang-II AT1 receptor and PPAR $\gamma$ are mutually antagonistic (40).

Although total PDE5 protein expression was not changed in either S-P467L mice or upon RhoBTB1 restoration, both PDE5 activity and p-PDE5 levels were elevated in S-P467L aorta and normalized upon RhoBTB1 restoration. A detailed mechanism of how RhoBTB1 regulates PDE5 activity is still under investigation. Most BTB domain-containing CRL3 substrate adaptors are associated with substrate proteolysis, mainly through K48-linked polyubiquitination (41). Thus, it is possible that RhoBTB1 might increase PDE5 activity by targeting a small portion of PDE5 proteins that are activated. Since the affinity of RhoBTB1 for PDE5 (Ser92A) is no higher than that of WT, it is possible that RhoBTB1 is recognizing other activation markers on PDE5. There is emerging evidence indicating a nondegradative role of CRL3 mediated by monoubiquitination. However, the continuous smear of PDE5 ubiquitination suggests that polyubiquitination is more likely.

It is notable that RhoBTB1 very effectively reversed NO resistance but had no impact on the enhanced vascular constriction induced by several agonists and failed to reduce increased RhoA and ROCK activity. Consistent with this, RhoBTB1 and RhoA did not coimmunoprecipitate in HEK293 cells (data not shown), further suggesting that RhoA/ROCK is not a direct target of RhoBTB1. Thus, this work demonstrates that PPAR $\gamma$ promotes at least 2 separable effects: (a) PPAR $\gamma$ reduces vasoconstriction by restraining RhoA/ROCK activity and (b) promotes vasorelaxation by restraining PDE5 through the RhoBTB1/Cullin-3 pathway. One of the most notable findings of the study was that RhoBTB1 could rapidly reverse established arterial stiffness. Arterial stiffness was also reversed by inhibition of PDE5 and was reestablished when PDE5 inhibition was terminated. These data support the concept that hypertension and arterial stiffness are related to mechanisms that control the vasodilator arm rather than the vasoconstrictor arm of the pathway (see model in Figure $8 \mathrm{~N}$ ). The data further suggest that the bioavailability of cGMP plays a fundamental role as a determinant of arterial distensibility and stiffness, major factors related to cardiovascular disease in the elderly $(31,42)$.

PDE5 inhibitors (avanafil, sildenafil, tadalafil, and vardenafil) are clinically used for the treating male erectile dysfunction but also have beneficial effects on vascular disease, coronary artery disease, hypertension, and diabetes (43). PDE5 inhibition is approved as a treatment for pulmonary hypertension and significantly reduces BP in patients with essential hypertension. Our data show that tadalafil treatment did not change BP or PWV in control mice, which was consistent with previous studies showing that this treatment did not significantly lower BP in healthy subjects (44). This could be interpreted to mean that PDE5 activity is low under normal conditions. We showed that PDE activity is elevated during 
hypertension caused by PPAR $\gamma$ dysfunction in SMCs and that this dysfunction can be reversed by direct inhibitors of PDE5 or by RhoBTB1 replacement. This mechanism may also contribute to other causes of hypertension, as suggested by the reduced expression of RhoBTB1 in response to Ang-II and the protection from Ang-II-induced hypertension mediated by SMC-specific reexpression of RhoBTB1. It is notable that RhoBTB1 only partially attenuated the pressor response to Ang-II. This is not surprising, as the effects of Ang-II are not tissue specific, whereas the effects of RhoBTB1 were specifically targeted to vascular smooth muscle. Indeed, Ang-II can act at the level of the brain to increase sympathetic outflow and the kidneys to cause fluid and salt retention, which are both pro-hypertensive effects $(45,46)$. Ang-II also activates the immune system, leading to inflammation in hypertension (47). Therefore, the RhoBTB1 pathway may be an attractive therapeutic target in hypertension or in other conditions in which vascular dysfunction is a result of reduced RhoBTB1 expression. Thus, studies are warranted to determine whether RhoBTB1 improves vascular function and $\mathrm{BP}$ in diabetes and atherosclerosis.

RhoBTB1 was first identified as a candidate tumor suppressor gene $(48,49)$ and was recently reported to play a role in cancer cell invasion by regulating the integrity of the Golgi apparatus (50), but, to our knowledge, no targets of RhoBTB1 have been identified until now. Our findings suggest that one of the functions of RhoBTB1 is to regulate PDE5 activity. RhoBTB1-mediated regulation of PDE5 might extend beyond the vasculature. For example, targeting PDE5 might explain the linkage between RhoBTB1 and cancer, since PDE5 inhibition reduces rat urinary bladder tumorigenesis (51).

To place this in perspective, the PROactive trial reported that pioglitazone decreased macrovascular events and lowered $\mathrm{BP}$ and cardiovascular risk (2). Although TZDs are an effective medication for type 2 diabetes, TZD treatment is associated with adverse effects such as weight gain, congestive heart failure, and increased bone fracture risk (52). Thus, identifying the molecular targets of PPAR $\gamma$ will help lead to the design of a new class of therapeutics that regulate PPAR $\gamma$ downstream actions more selectively. This study shows that a PPAR $\gamma$ target gene, RhoBTB1, mediates PPAR $\gamma$-mediated regulation of BP and vascular homeostasis by regulating PDE5 activity through a Cullin-3-dependent mechanism. Our study further suggests that, in conditions in which PPAR $\gamma$ activity is impaired, such as in obesity and diabetes, PDE5 inhibition might provide cardiovascular protection.

\section{Methods}

Generation and validation of RhoBTB1 ${ }^{I N D}$. Full-length RhoBTB1 was amplified from heart cDNA from C57BL/6J mice using the primers 5'-GCATGAACTAGTATGGACTCTGACATGGACTACGAACGAC-3' (forward) and 5'-CATGAACTAGTTCAGGCGACAGCTGGGGACGAAT-3' (reverse), with SpeI restriction enzyme sites inserted. RhoBTB1 cDNA was digested with SpeI and then subcloned into a previously described vector carrying the CAG promoter/enhancer, the loxP-STOP-loxP sequence, and IRES-tdTomato $(53,54)$. The construct was sequenced to confirm RhoBTB1 insertion and determine its orientation. The function of the RhoBTB1 ${ }^{\mathrm{IND}}$ construct was tested in HEK293T cell lines. HEK293T cells plated in 6-well dishes were transfected using Lipofectamine LTX (Invitrogen, Thermo Fisher Scientific) according to the manufacturer's protocol with either a Myc-
RhoBTB1 or RhoBTB1 ${ }^{\text {IND }}$ construct with or without a plasmid encoding Cre recombinase ( $\mathrm{p} 185-\mathrm{Cre})$. Control transfections contained the inducible construct backbone with or without p185-Cre. The cells were lysed for Western blotting 48 hours after transfection.

The RhoBTB1 ${ }^{\text {IND }}$ transgene was excised from the vector backbone and microinjected into pronuclei of B6SJL mice (C57BL/6J SJL/J) to generate founders. The transgenic mice were maintained by backcross breeding with C57BL/6J mice for at least 4 generations. Genomic DNA was isolated from mouse tail biopsies, and genotyping was performed by PCR using the primers 5'-ACCACTACCAGCGTGTGAAGA-3' and 5'-GCGCATGAACTCTTTGATGACCT-3'. Double-transgenic mice were produced by crossing the RhoBTB1 ${ }^{\mathrm{IND}}$ transgenic mice with SMCCreER $^{\mathrm{T} 2}$ mice expressing Tx-inducible Cre recombinase $\left(\mathrm{CreER}^{\mathrm{T} 2}\right)$ under the control of the smooth muscle myosin heavy chain (SMMHC) promoter [B6.FVB-Tg(Myh11-cre/ERT2)1Soff/J, The Jackson Laboratory, stock 019079]. The final experimental triple-transgenic mice (S-P467L S-RhoBTB1) were obtained by crossing the double-transgenic mice with S-P467L transgenic mice carrying dominant-negative P467L PPAR $\gamma$ under the control of the SMMHC promoter, as described previously (16). To induce expression of RhoBTB1, Tx-free base (MilliporeSigma, T5648) was dissolved in corn oil (MilliporeSigma, C8267) at a concentration of $20 \mathrm{mg} / \mathrm{ml}$ by shaking overnight at $37^{\circ} \mathrm{C}$. Tx $(75$ $\mathrm{mg} / \mathrm{kg} \mathrm{BW}$ ) was administered by i.p. injection on 5 consecutive days.

We generated S-ROSA-transgenic mice expressing the tdTomato reporter in smooth muscle by crossing ROSA26 reporter mice with mice expressing a Tx-inducible Cre recombinase (CreERT2) under the control of the SMMHC promoter.

Western blot and IP analyses. Western blotting was performed using transfected HEK293 cells or aortic tissue as previously described (19, 20). Lipofectamine (Thermo Fisher Scientific) was used to transfect HEK293 cells. The total amount of DNA was always held constant. For ubiquitination assays, cells were treated with MG132 (10 $\mu \mathrm{mol} / \mathrm{l}$ for 2 hours) and/or MLN4924 ( $1 \mu \mathrm{mol} / \mathrm{l}$ for 16 hours before lysis). Protein was extracted in a lysis buffer containing $50 \mathrm{mmol} / \mathrm{l}$ Tris $\mathrm{Cl}, 0.1 \mathrm{mmol} / \mathrm{l}$ EDTA (pH 7.5), 0.1 mmol/1 EGTA, $1 \%$ w/v sodium deoxycholic acid, $1 \%$ $\mathrm{w} / \mathrm{v}$ NP-40, and $0.1 \% \mathrm{w} / \mathrm{v}$ SDS, with a protease inhibitor (Roche) and phosphatase inhibitors (Roche). SDS was removed for co-IP experiments. Deubiquitinase (DUB) inhibitors ( $25 \mathrm{mmol} / \mathrm{l} \mathrm{N}$-ethylmaleimide) were added in the lysis buffer for the ubiquitination assays. Samples were centrifuged $(12,000 \mathrm{~g})$ for 10 minutes at $4^{\circ} \mathrm{C}$, and supernatants were collected. The protein concentration in the lysates was determined by Lowry assay (Bio-Rad). For co-IP experiments, $0.5-1.5 \mathrm{mg}$ total protein lysate was incubated with $20 \mu \mathrm{l} \mathrm{Myc} \mathrm{or} \mathrm{His} \mathrm{AC} \mathrm{antibody} \mathrm{(Santa} \mathrm{Cruz}$ Biotechnology, sc-98036) for 2 hours or overnight at $4^{\circ} \mathrm{C}$. Protein $(0.2$ $\mathrm{mg}$ ) was used for the ubiquitination assays. Beads were washed 4 times with lysis buffer, and immunoprecipitates were eluted for 10 minutes at $100^{\circ} \mathrm{C}$ in $50 \mu \mathrm{l}$ of $2 \times$ sample buffer. Precipitated proteins or equal amounts of cell or tissue lysates $(10-1000 \mu \mathrm{g})$ were separated by SDSPAGE (7\%-15\%) and transferred onto a nitrocellulose membranes (GE Healthcare). After blocking with 5\% nonfat milk, the membranes were incubated with primary antibodies at $4^{\circ} \mathrm{C}$ overnight and then visualized using HRP-conjugated secondary antibodies (GE Healthcare, 1:10,000 dilution, at room temperature for $1 \mathrm{~h}$ ) for Western blotting and EasyBlot anti-rabbit IgG (GeneTex, GTX221666-01: 1:2000, at room temperature for $1 \mathrm{~h}$ ) for IP experiments. RhoBTB1 (Abcam, ab59123), tdTomato (Gene Tex, GTX127897), Myc (Cell Signaling Technology, 2435), PPAR $\gamma$ (Cell Signaling Technology, 2435), HA (eBioscience, 14-6756), His 
(Cell Signaling Technology, 2365), Cullin-3 (Bethyl Laboratories, A301109A), p-MYPT1 (Thr696) (Cell Signaling Technology, 5163), sGC $\alpha 1$ (Abcam, ab50358), sGC $\beta 1$ (Abcam, ab154841), PKG1 (Cell Signaling Technology, 3248), p-PDE5 (GeneTex, GTX36930), and PDE5 (Cell Signaling Technology, 2395) were used for these studies. GAPDH (Santa Cruz Biotechnology, sc-32233) was used as a loading control.

Co-IP. Plasmids were transfected into HEK293 cells using Lipofectamine LTX with Plus Reagent (Thermo Fisher Scientific, 15338100). After 24 hours, cells were treated with the proteasome inhibitor MG132 (10 $\mu \mathrm{M}, 2 \mathrm{~h}$ ) before protein isolation. Protein (1 mg) from cell lysates was incubated with $20 \mu \mathrm{l}$ anti-c-Myc (Santa Cruz Biotechnology, sc-40) or anti-His agarose beads (Santa Cruz Biotechnology, sc-8038) for 4 hours at $4^{\circ} \mathrm{C}$. Beads were washed 4 times $(1500$ rcf, $4^{\circ} \mathrm{C}, 5 \mathrm{~min}$ ) and boiled with $2 \times$ loading buffer at $95^{\circ} \mathrm{C}$ for $5 \mathrm{~min}$ utes to release bound protein. Precipitated protein was collected by centrifugation (2000 rcf, 2 min), separated by $8 \%$ SDS-PAGE gel, and transferred onto a PVDF membrane (Immobolin) at $35 \mathrm{mV}$ overnight at $4^{\circ} \mathrm{C}$. Primary antibodies against PDE5 (Cell Signaling Technology, 2395), RhoBTB1 (Abcam, ab22022), and GAPDH (Santa Cruz Biotechnology, sc-47724) were used to detect target proteins.

Real-time RT-PCR. RNA was extracted from HEK293T cells or aortic tissue, and real-time RT-PCR (qPCR) was performed as previously described (55). Briefly, using oligo (dT) primers, RNaseOUT (Invitrogen, Thermo Fisher Scientific), and SuperScript III (Invitrogen, Thermo Fisher Scientific), cDNA was synthesized from 400 to 700 ng total RNA extracted using RNeasy spin columns (RNeasy Mini Kit, QIAGEN). Each qPCR reaction was performed in duplicate. cDNA (10 ng) was subjected to TaqMan Gene Expression assays using TaqMan Fast Advanced Master Mix (Applied Biosystems) and the targeted TaqMan probes. The following TaqMan probes were used on an Applied Biosystems StepOnePlus system to evaluate gene expression levels: mouse GAPDH (4352932-0905028), mouse RhoBTB1 (Mm01143659_m1), and human $\operatorname{PPAR} \gamma$ (Hs01115513_m1). In some experiments, qPCR reactions were performed with $10 \mathrm{ng}$ cDNA and Fast SYBR Green Master Mix (Applied Biosystems) along with the targeted gene primers in a total volume of $10 \mu \mathrm{l}$. The primers used for tdTomato were: (forward) 5'-CGAGGAGGTCATCAAAGAGTTC-3' and (reverse) 5'-GGGAAGGACAGCTTCTTGTAAT-3'. $\triangle \triangle$ Ct values were calculated using GAPDH as a reference gene to determine relative mRNA expression levels.

Immunostaining. Cells were fixed with $4 \%$ paraformaldehyde at room temperature for 20 minutes. Following this incubation, cells were rinsed 3 times with PBS. TdTomato expression was determined using polyclonal anti-tdTomato (LS-C340696, LifeSpan BioSciences), and the endothelium was identified with vWF antibody (ab8822, Abcam) diluted in $5 \%$ normal goat serum with $0.1 \%$ Triton X-100 to a final dilution of $1: 250$ and then incubated at $4^{\circ} \mathrm{C}$ overnight. The cells were then rinsed in TBS-T (TBS with 0.3\% Tween-20) 3 times for 10 minutes. The secondary antibody Alexa Fluor 488 (Abcam) was diluted in 5\% goat serum, $0.1 \%$ Triton X-100 in TBS to a final dilution of 1:1000 and incubated at room temperature for 1 hour. All incubation and rinsing steps were performed under constant agitation. The cells were mounted with VECTASHIELD (Vector Laboratories) and imaged for the presence of green fluorescence on a Zeiss LSM710 confocal microscope. Single-plane images were collected. When comparing detection of transgene expression between samples, we kept the microscope settings, including laser power, gain, and offset, constant throughout image collection process. Final images were processed using ImageJ soft- ware, version 1.48 (NIH; Java, 1.6.0-20, 64-bit) to make adjustments to image size or linear parameters such as brightness and contrast. All adjustments were kept consistent across samples.

Measurement of BP and heart rate. BP was recorded by radiotelemetry (16). Mice were anesthetized with ketamine and xylazine, and a radiotelemetric BP probe (TA11PA-C10, DSI) was implanted into the common carotid artery. The mice were allowed to recover for 7 days. $\mathrm{BP}$ was recorded for 10 seconds every 5 minutes on 7 consecutive days. Data from each animal were averaged hourly, and corresponding times across the 7 days were averaged for each animal to create a single composite 24-hour tracing for each animal.

Vascular function. Aortic function was assessed using a wire myograph preparation as previously described (20). The thoracic aorta was dissected free of perivascular fat and cut into 4 segments. Aortic rings were then equilibrated for 45 minutes under a resting tension of $0.5 \mathrm{~g}$, and vasoconstriction was recorded in response to $\mathrm{KCl}(10-100$ $\mathrm{mM})$. Concentration-dependent response curves to ACh $(1 \mathrm{nM}-30 \mu \mathrm{M})$, SNP (0.1 nM-30 $\mu \mathrm{M})$, BAY41-2272 (0.1 nM-30 $\mu \mathrm{M}), 8$-Br-cGMP (100 nM-300 $\mu \mathrm{M})$, 8-pCPT-cGMP (100 nM-100 $\mu \mathrm{M})$, or NS1619 (10 nM-30 $\mu \mathrm{M})$ were performed after an initial submaximal precontraction $(40 \%-$ $60 \%)$ with PGF $2 \alpha(3-10 \mu \mathrm{M})$. In a separate set of experiments, contraction was recorded for 5-HT (10 nM-10 $\mu \mathrm{M})$ and ET-1 (0.1-30 nM).

Basilar arteries were isolated and cannulated onto glass micropipettes filled with oxygenated Krebs buffer (118.3 mmol/l NaCl, $4.7 \mathrm{mmol} / \mathrm{l} \mathrm{KCl}, 1.2 \mathrm{mmol} / 1 \mathrm{MgSO}_{4}, 1.2 \mathrm{mmol} / 1 \mathrm{KH}_{2} \mathrm{PO}_{4}, 25 \mathrm{mmol} / \mathrm{l}$ $\mathrm{NaHCO}_{3}, 2.5 \mathrm{mmol} / \mathrm{l} \mathrm{CaCl}_{2}$, and $11 \mathrm{mmol} / \mathrm{l}$ glucose) in an organ chamber as we reported previously $(21,22)$. Arteries were transferred to a pressurized myograph system (DMT) and equilibrated for 30 minutes at $60 \mathrm{mmHg}$ under no-flow conditions, and viability was first examined by exposure to $\mathrm{KCl}(100 \mathrm{mM})$. Relaxation was recorded for ACh $(1 \mathrm{nM}-100 \mu \mathrm{M})$ after initial preconstriction by $30 \%$ with U46619, a thromboxane A2 mimetic. The level of preconstriction was similar in nontransgenic and S-P467L mice.

Arterial stiffness and compliance. PWV was measured using the transit time method with Doppler ultrasound (Mouse Doppler, Indus Instruments) (30). Mice anesthetized with isoflurane were placed in supine positions on a heated platform $\left(38^{\circ} \mathrm{C}\right)$, with ECG electrodes taped to their paws for continuous recordings. Pressure waveforms using a $20-\mathrm{MHz}$ probe were imaged at the descending aorta and abdominal aorta, $1 \mathrm{~mm}$ above the exit to the left renal artery. Arrival and transit times were calculated over 5 cardiac cycles, and the distance between the descending and abdominal aortae was measured using a ruler. PWV $(\mathrm{m} / \mathrm{s})$ was calculated by dividing the distance by the transit time. Masson's trichrome staining and a hydroxyproline assay were used to measure aortic collagen (56).

Distensibility was calculated using the equation below, where $d$ is the vessel outer diameter, and "sys" and "dias" denote systolic and diastolic values of diameter and pressure as previously described (57).

$$
\mathrm{D}=\left[d_{\text {sys }}-d_{\text {dias }}\right] /\left[\left(d_{\text {dias }}\right)\left(P_{\text {sys }}-P_{\text {dias }}\right)\right]
$$

\section{(Equation 1)}

The 24-hour mean systolic and diastolic BP values for each genotype group after $T x$ were used to calculate $d_{\text {sys }}$ and $d_{\text {dias }}$ via linear interpolation using the pressure-diameter data for each mouse.

Pressure-diameter curves were used to measure arterial compliance. Segments of the thoracic aorta were mounted on $0.7-\mathrm{mm}$ can- 
nulas and extended to the in situ length. Vessels were placed in calcium-free buffer to eliminate active tone. Intraluminal pressure was increased in a step-wise fashion, while video microscopy was used to follow outer and inner diameters. Diameters were recorded with each increment of $25 \mathrm{mmHg}$ from 0 to $200 \mathrm{mmHg}$. The maximal intraluminal pressure was $200 \mathrm{mmHg}$, because both the lumen and outer diameters reach a plateau beyond this pressure. Pressure-diameter curves were constructed for the calculation of vascular compliance, in which the increment of vessel diameter from $0 \mathrm{mmHg}$ was plotted against the pressure. The traditional stress-strain relationship was determined as described previously (58).

cGMP and PDE activity. cGMP concentration in aortae from mice was measured using an ELISA kit (Cyclic GMP EIA Ki, Cayman Chemical) according to the manufacturer's specifications. PDE activity in aortae from mice was measured using a PDE Activity Assay Kit (Colorimetric, ab139460).

Site-directed mutagenesis. To obtain a PDE5 mutation (Ser92 Ala), site-directed mutagenesis was performed on Myc-PDE5 using a PCRbased strategy with the QuikChange Site-directed Mutagenesis Kit (Agilent Technologies). Briefly, a mutagenic primer was designed using the Agilent QuikChange primer design program. The primer was designed from PDE5 (WT) coding sequence cDNA, with changes of $\mathrm{T}$ to $\mathrm{G}$ at nucleotide 274 . This mutagenesis did not alter the original protein-coding sequence of PDE5, which was verified by sequencing.

Enzymatic activity of PDE5 was measured according to the instructions of the Colorimetric PDE Activity Assay Kit (Abcam, ab139460). Recombinant human PDE5 (31.2 ng) (Enzo, ENZ-PRT126-0010) was incubated with $20 \mu \mathrm{l} 5^{\prime}$-cGMP $(0.5 \mu \mathrm{M})$ and $10 \mu \mathrm{l} 5^{\prime}$-nucleotidase under $30^{\circ} \mathrm{C}$ for 105 minutes. The PDE5 inhibitor tadalafil $(2 \mu \mathrm{M})$ (Selleckchem, S1512) was used as a positive control. Desalted recombinant human RhoBTB1 (31.2 ng or $312 \mathrm{ng}$ ) (Novus Biologicals, H00009886-P01-2 $\mu \mathrm{g})$ was incubated with PDE5 to achieve a 1:1 or 1:10 molar ratio. The reaction was terminated by incubation with termination buffer under room temperature for 20 minutes. $\mathrm{OD}_{620 \mathrm{~nm}}$ were measured on a plate reader to determine the amount of GMP generated.

Chemicals. ACh, SNP, KCl, 5-HT, ET-1, L-NAME, and human Ang-II (A9525) were obtained from MilliporeSigma. PGF2 $\alpha$ (Pfizer), Y-27632 (Calbiochem), 8-Br-cGMP (Tocris), and BAY41-2722 (Cayman) were also used. MLN4924 (Active-Biochem), Zaprinast (Tocris), MBCQ (Tocris), and tadalafil (Selleckchem) for ex vivo studies were dissolved in DMSO. NS1619 (Tocris) and Cilostamide (Cayman) were dissolved in ethanol. Tadalafil for in vivo studies was dissolved in $30 \%$ propylene glycol, $5 \%$ Tween- 80 and $65 \%$ (5\% dextrose) in water according to the manufacturer's instructions.

Statistics. Experiments were performed in similar numbers using both male and female mice. There was no difference between male and female mice, and therefore all data were merged. Results are expressed as the mean \pm SEM. Statistical evaluation of the data was performed using GraphPad Prism software. Where appropriate, a paired or unpaired, 2-tailed Student's $t$ test was used to compare between 2 groups. In other studies, ANOVA followed by Tukey's test for comparisons was performed. A $P$ value of less than 0.05 was considered significant.
Study approval. The mice were housed in the University of Iowa Transgenic Facility and fed standard mouse chow and water ad libitum. Care of the mice met NIH guidelines and standards, and all experiments were approved by the IACUCs of the University of Iowa and the Medical College of Wisconsin.

Requests for materials. All requests for materials should be directed to Curt D. Sigmund, Medical College of Wisconsin, Milwaukee, Wisconsin.

\section{Author contributions}

MM conceived the research and conducted many of the experiments. SF performed co-IP, ubiquitination, PDE5 mutagenesis, and PDE5 activity assays. JW performed studies of arterial stiffness by PWV and calculations of distensibility. LNA performed vascular reactivity experiments on the cerebral basilar artery. ARN assisted with studies of vascular reactivity. SRCI developed and performed the initial characterization the RhoBTB1-inducible transgenic mouse model. $\mathrm{CH}$ performed vascular reactivity experiments on the cerebral basilar artery. XL performed co-IP and ubiquitination studies. KTL conducted general molecular biology and vascular reactivity studies. DFG performed immunohistochemical analyses for tdTomato and the endothelial marker gene. DRD performed surgeries for implantation of the BP radiotelemeters. HLK conducted analysis of transcriptomic data leading to identification of RhoBTB1 and extensive revisions to the early draft of the manuscript. FWQ contributed extensive scientific discussions and co-mentored all trainee authors on this manuscript. CDS coordinated all funding and studies and provided extensive revisions and commentary on the manuscript.

\section{Acknowledgments}

The authors thank Bill Paradee, Norma Sinclair, JoAnne Schwarting, and Patricia Yarolem from the University of Iowa for generating and genotyping the mice used in this study. Transgenic mice were generated at the University of Iowa Genome Editing Facility, which is supported in part by grants from the NIH (HL084207) and from the Roy J. and Lucille A. Carver College of Medicine. We would like to thank Chantal Allamargot (University of Iowa) for assistance with Masson's trichrome staining. We also acknowledge use of equipment and assistance from the Central Microscopy Facility at the University of Iowa. This work was supported through research grants from the NIH (HL084207, HL125603, HL131689, and HL144807 to CDS), the American Heart Association (15SFRN23480000, to CDS and 17POST33660685, to JW), and the University of Iowa. The authors gratefully acknowledge the generous research support of the Roy J. Carver Trust. The contents of this manuscript are solely the responsibility of the authors and do not necessarily represent the official views of the University of Iowa.

Address correspondence to: Curt D. Sigmund, Department of Physiology, Medical College of Wisconsin, 8701 Watertown Plank Road, Milwaukee, Wisconsin 53226-0509, USA. Phone: 414.955.8277; Email: csigmund@mcw.edu.
1. Lehmann JM, Moore LB, Smith-Oliver TA, Wilkison WO, Willson TM, Kliewer SA. An antidiabetic thiazolidinedione is a high affinity ligand for peroxisome proliferator-activated receptor gamma (PPAR gamma). J Biol Chem. 1995;270(22):12953-12956.

2. Dormandy JA, et al. Secondary prevention of macrovascular events in patients with type 2 diabetes in the PROactive study (PROspective pioglitAzone Clinical Trial In macroVascular Events): a randomised controlled trial. Lancet 2005;366(9493):1279-1289. 
3. Ogihara T, Rakugi H, Ikegami H, Mikami H, Masuo K. Enhancement of insulin sensitivity by troglitazone lowers blood pressure in diabetic hypertensives. Am J Hypertens. 1995;8(3):316-320.

4. Sung BH, Izzo JL, Dandona P, Wilson MF. Vasodilatory effects of troglitazone improve blood pressure at rest and during mental stress in type 2 diabetes mellitus. Hypertension. 1999;34(1):83-88.

5. Bennett SM, et al. Rosiglitazone improves insulin sensitivity, glucose tolerance and ambulatory blood pressure in subjects with impaired glucose tolerance. Diabet Med. 2004;21(5):415-422.

6. Nissen SE, Wolski K. Effect of rosiglitazone on the risk of myocardial infarction and death from cardiovascular causes. $N$ Engl J Med. 2007;356(24):2457-2471.

7. Mitka M. FDA eases restrictions on the glucose-lowering drug rosiglitazone. JAMA. 2013;310(24):2604

8. Home PD, et al. Rosiglitazone evaluated for cardiovascular outcomes in oral agent combination therapy for type 2 diabetes (RECORD): a multicentre, randomised, open-label trial. Lancet. 2009;373(9681):2125-2135.

9. Hashim S, Gomes T, Juurlink D, Hellings C, Mamdani M. The rise and fall of the thiazolidinediones: impact of clinical evidence publication and formulary change on the prescription incidence of thiazolidinediones. J Popul Ther Clin Pharmacol. 2013;20(3):e238-e242.

10. Diep QN, et al. Structure, endothelial function, cell growth, and inflammation in blood vessels of angiotensin II-infused rats: role of peroxisome proliferator-activated receptor-gamma. Circulation. 2002;105(19):2296-2302.

11. Barroso I, et al. Dominant negative mutations in human PPARgamma associated with severe insulin resistance, diabetes mellitus and hypertension. Nature. 1999;402(6764):880-883.

12. Kis A, et al. Defective peroxisomal proliferators activated receptor gamma activity due to dominant-negative mutation synergizes with hypertension to accelerate cardiac fibrosis in mice. Eur J Heart Fail. 2009;11(6):533-541.

13. Auclair M, et al. Peroxisome proliferator-activated receptor- $\gamma$ mutations responsible for lipodystrophy with severe hypertension activate the cellular renin-angiotensin system. Arterioscler Thromb Vasc Biol. 2013;33(4):829-838.

14. Savage DB, et al. Human metabolic syndrome resulting from dominant-negative mutations in the nuclear receptor peroxisome proliferator-activated receptor-gamma. Diabetes. 2003;52(4):910-917.

15. Buchanan TA, et al. Blood pressure lowering by pioglitazone. Evidence for a direct vascular effect. J Clin Invest. 1995;96(1):354-360.

16. Halabi CM, et al. Interference with PPAR gamma function in smooth muscle causes vascular dysfunction and hypertension. Cell Metab. 2008;7(3):215-226.

17. Beyer AM, et al. Interference with PPARgamma signaling causes cerebral vascular dysfunction, hypertrophy, and remodeling. Hypertension. 2008;51(4):867-871.

18. Beyer AM, et al. Endothelium-specific interference with peroxisome proliferator activated receptor gamma causes cerebral vascular dys- function in response to a high-fat diet. Circ Res. 2008;103(6):654-661.

19. Mukohda M, et al. Hypertension-causing mutation in peroxisome proliferator-activated receptor $\gamma$ impairs nuclear export of nuclear factor- $\mathrm{kB}$ p65 in vascular smooth muscle. Hypertension. 2017;70(1):174-182.

20. Mukohda M, Stump M, Ketsawatsomkron P, Hu C, Quelle FW, Sigmund CD. Endothelial PPAR- $\gamma$ provides vascular protection from IL-1 $\beta$-induced oxidative stress. Am J Physiol Heart Circ Physiol. 2016;310(1):H39-H48.

21. $\mathrm{Hu} \mathrm{C}$, et al. Retinol-binding protein 7 is an endothelium-specific PPAR. JCI Insight. 2017;2(6):e91738.

22. Hu C, Lu KT, Mukohda M, Davis DR, Faraci FM, Sigmund CD. Interference with PPAR $\gamma$ in endothelium accelerates angiotensin II-induced endothelial dysfunction. Physiol Genomics. 2016;48(2):124-134.

23. Pelham CJ, et al. Cullin-3 regulates vascular smooth muscle function and arterial blood pressure via PPAR $\gamma$ and RhoA/Rho-kinase. Cell Metab. 2012;16(4):462-472.

24. Berthold J, Schenkova K, Rivero F. Rho GTPases of the RhoBTB subfamily and tumorigenesis. Acta Pharmacol Sin. 2008;29(3):285-295.

25. Geyer R, Wee S, Anderson S, Yates J, Wolf DA. $\mathrm{BTB} / \mathrm{POZ}$ domain proteins are putative substrate adaptors for cullin 3 ubiquitin ligases. Mol Cell. 2003;12(3):783-790.

26. Berthold J, et al. Characterization of RhoBTBdependent Cul3 ubiquitin ligase complexes--evidence for an autoregulatory mechanism. Exp Cell Res. 2008;314(19):3453-3465.

27. Chen Y, et al. Cullin mediates degradation of RhoA through evolutionarily conserved BTB adaptors to control actin cytoskeleton structure and cell movement. Mol Cell. 2009;35(6):841-855.

28. Ibeawuchi SR, Agbor LN, Quelle FW, Sigmund CD. Hypertension-causing mutations in Cullin3 protein impair RhoA protein ubiquitination and augment the association with substrate adaptors. J Biol Chem. 2015;290(31):19208-19217.

29. Boyden LM, et al. Mutations in kelch-like 3 and cullin 3 cause hypertension and electrolyte abnormalities. Nature. 2012;482(7383):98-102.

30. Agbor LN, et al. Cullin-3 mutation causes arterial stiffness and hypertension through a vascular smooth muscle mechanism. JCI Insight. 2016;1(19):e91015.

31. O'Rourke MF. Isolated systolic hypertension, pulse pressure, and arterial stiffness as risk factors for cardiovascular disease. Curr Hypertens Rep. 1999;1(3):204-211.

32. Rybalkin SD, Yan C, Bornfeldt KE, Beavo JA. Cyclic GMP phosphodiesterases and regulation of smooth muscle function. Circ Res. 2003;93(4):280-291.

33. Corbin JD, Turko IV, Beasley A, Francis SH. Phosphorylation of phosphodiesterase- 5 by cyclic nucleotide-dependent protein kinase alters its catalytic and allosteric cGMP-binding activities. Eur JBiochem. 2000;267(9):2760-2767.

34. Rybalkin SD, Rybalkina IG, Feil R, Hofmann F, Beavo JA. Regulation of cGMP-specific phosphodiesterase (PDE5) phosphorylation in smooth muscle cells. J Biol Chem. 2002;277(5):3310-3317.
35. He W, et al. Adipose-specific peroxisome proliferator-activated receptor gamma knockout causes insulin resistance in fat and liver but not in muscle. Proc Natl Acad Sci US A. 2003;100(26):15712-15717.

36. Keen HL, et al. Bioinformatic analysis of gene sets regulated by ligand-activated and dominant-negative peroxisome proliferator-activated receptor gamma in mouse aorta. Arterioscler Thromb Vasc Biol. 2010;30(3):518-525.

37. Keen HL, et al. Gene expression profiling of potential PPARgamma target genes in mouse aorta. Physiol Genomics. 2004;18(1):33-42.

38. Matsuda T, Yanase S, Takaoka A, Maruyama M. The immunosenescence-related gene Zizimin2 is associated with early bone marrow B cell development and marginal zone B cell formation. Immun Ageing. 2015;12:1.

39. Mota de Sá P, Richard AJ, Hang H, Stephens JM. Transcriptional regulation of adipogenesis. Compr Physiol. 2017;7(2):635-674.

40. Sigmund CD. A clinical link between peroxisome proliferator-activated receptor $\gamma$ and the renin-angiotensin system. Arterioscler Thromb Vasc Biol. 2013;33(4):676-678.

41. Genschik P, Sumara I, Lechner E. The emerging family of CULLIN3-RING ubiquitin ligases (CRL3s): cellular functions and disease implications. EMBO J. 2013;32(17):2307-2320.

42. Wallace SM, et al. Isolated systolic hypertension is characterized by increased aortic stiffness and endothelial dysfunction. Hypertension. 2007;50(1):228-233.

43. Ghiadoni L, Versari D, Taddei S. Phosphodiester ase 5 inhibition in essential hypertension. Curr Hypertens Rep. 2008;10(1):52-57.

44. Kloner RA, Mitchell M, Emmick JT. Cardiovascular effects of tadalafil. Am J Cardiol. 2003;92(9A):37M-46M.

45. Nakagawa P, Sigmund CD. How is the brain renin-angiotensin system regulated? Hypertension. 2017;70(1):10-18.

46. Coffman TM. The inextricable role of the kidney in hypertension. J Clin Invest. 2014;124(6):2341-2347.

47. Guzik TJ, et al. Role of the T cell in the genesis of angiotensin II induced hypertension and vascular dysfunction. J Exp Med. 2007;204(10):2449-2460.

48. Beder LB, et al. Identification of a candidate tumor suppressor gene RHOBTB1 located at a novel allelic loss region $10 \mathrm{q} 21$ in head and neck cancer. JCancer Res Clin Oncol. 2006;132(1):19-27.

49. Beder LB, et al. Genome-wide analyses on loss of heterozygosity in head and neck squamous cell carcinomas. Lab Invest. 2003;83(1):99-105.

50. McKinnon CM, Mellor H. The tumor suppressor RhoBTB1 controls Golgi integrity and breast cancer cell invasion through METTL7B. BMC Cancer. 2017;17(1):145.

51. Piazza GA, et al. Exisulind, a novel proapoptotic drug, inhibits rat urinary bladder tumorigenesis. Cancer Res. 2001;61(10):3961-3968.

52. Ahmadian M, et al. PPAR $\gamma$ signaling and metabolism: the good, the bad and the future. Nat Med 2013;19(5):557-566.

53. Stump M, et al. Effect of selective expression of dominant-negative PPAR $\gamma$ in pro-opiomelanocortin neurons on the control of energy balance. Physiol Genomics. 2016;48(7):491-501. 
54. Stump M, et al. Nervous system expression of PPAR $\gamma$ and mutant PPAR $\gamma$ has profound effects on metabolic regulation and brain development. Endocrinology. 2016;157(11):4266-4275.

55. Lu KT, Keen HL, Weatherford ET, Sequeira-Lopez ML, Gomez RA, Sigmund CD. Estrogen receptor $\alpha$ is required for maintaining baseline renin expression. Hypertension. 2016;67(5):992-999.

56. $\mathrm{Wu}$ J, et al. Inflammation and mechanical stretch promote aortic stiffening in hypertension through activation of p38 mitogen-activated protein kinase. Circ Res. 2014;114(4):616-625.

57. Bersi MR, Bellini C, Wu J, Montaniel KRC, Harrison DG, Humphrey JD. Excessive adventitial remodeling leads to early aortic maladaptation in angiotensin-induced hypertension. Hypertension. 2016;67(5):890-896.

58. Hajdu MA, Heistad DD, Siems JE, Baumbach GL. Effects of aging on mechanics and composition of cerebral arterioles in rats. Circ Res. 1990;66(6):1747-1754. 